\title{
PERIOD INTEGRALS AND THE RIEMANN-HILBERT CORRESPONDENCE
}

\author{
AN HUANG, BONG H. LIAN, AND XINWEN ZHU
}

\begin{abstract}
A tautological system, introduced in 16] 17, arises as a regular holonomic system of partial differential equations that govern the period integrals of a family of complete intersections in a complex manifold $X$, equipped with a suitable Lie group action. A geometric formula for the holonomic rank of such a system was conjectured in [4, and was verified for the case of projective homogeneous space under an assumption. In this paper, we prove this conjecture in full generality. By means of the RiemannHilbert correspondence and Fourier transforms, we also generalize the rank formula to an arbitrary projective manifold with a group action.
\end{abstract}

\section{Contents}

1. Introduction

2. CY hyperplane sections

3. !-fibers of $\tau$

4. The geometry

5. An exact sequence for $\tau$

6. Rank 1 points of general $G / P$

7. Rank 1 points of 1 -step flags

8. Rank 1 points of $r$-step flags

Appendix A. Theory of $D$-modules

References

\section{INTRODUCTION}

Let $G$ be a connected algebraic group over a field $k$ of characteristic zero. Let $X$ be a projective $G$-variety and let $\mathcal{L}$ be a very ample $G$-linearized invertible sheaf over $X$ which gives rise to a $G$-equivariant embedding

$$
X \rightarrow \mathbb{P}(V),
$$

where $V=\Gamma(X, \mathcal{L})^{\vee}$. Let $r=\operatorname{dim} V$. We assume that the action of $G$ on $X$ is locally effective, i.e. $\operatorname{ker}(G \rightarrow \operatorname{Aut}(X))$ is finite. Let $\mathbb{G}_{m}$ be the multiplicative group acting on $V$ by homotheties. Let $\hat{G}=G \times \mathbb{G}_{m}$, whose Lie algebra $\hat{\mathfrak{g}}=\mathfrak{g} \oplus k e$, where $e$ acts on $V$ by identity. We denote by $Z: \hat{G} \rightarrow \mathrm{GL}(V)$ be the corresponding group representation, and $Z: \hat{\mathfrak{g}} \rightarrow \operatorname{End}(V)$ be the corresponding Lie algebra representation. In particular, $Z(e)$ is the Euler vector field on $V$. Note that under our assumptions, $Z: \hat{\mathfrak{g}} \rightarrow \operatorname{End}(V)$ is injective. 
Let $\hat{\imath}: \hat{X} \subset V$ be the cone of $X$, defined by the ideal $I(\hat{X})$. Let $\beta: \hat{\mathfrak{g}} \rightarrow k$ be a Lie algebra homomorphism. Then a tautological system as defined in [16] [17] is the cyclic D-module on $V^{\vee}$

$$
\tau(G, X, \mathcal{L}, \beta)=D_{V^{\vee}} / D_{V^{\vee}} J(\hat{X})+D_{V^{\vee}}(Z(\xi)+\beta(\xi), \xi \in \hat{\mathfrak{g}}),
$$

where

$$
J(\hat{X})=\{\widehat{D} \mid D \in I(\hat{X})\}
$$

is the ideal of the commutative subalgebra $\mathbb{C}[\partial] \subset D_{V^{\vee}}$ obtained by the Fourier transform of $I(\hat{X})$ (see $\$$ for the review of the Fourier transform and in particular (A.6) for the notation).

We recall the main motivation for studying tautological systems. Let $X^{\prime}$ be a compact complex manifold (not necessarily algebraic), such that the complete linear system of anticanonical divisors in $X^{\prime}$ is base point free. Let $\pi: \mathcal{Y} \rightarrow B:=\Gamma\left(X^{\prime}, \omega_{X^{\prime}}^{-1}\right)_{s m}$ be the family of smooth CY hyperplane sections $Y_{a} \subset X^{\prime}$, and let $\mathbb{H}^{\text {top }}$ be the Hodge bundle over $B$ whose fiber at $a \in B$ is the line $\Gamma\left(Y_{a}, \omega_{Y_{a}}\right) \subset H^{n-1}\left(Y_{a}\right)$, where $n=\operatorname{dim} X^{\prime}$. In [17, the period integrals of this family are constructed by giving a canonical trivialization of $\mathbb{H}^{\text {top }}$. Let $\Pi=\Pi\left(X^{\prime}\right)$ be the period sheaf of this family, i.e. the locally constant sheaf generated by the period integrals (Definition 1.1 [17].)

Let $V=\Gamma\left(X^{\prime}, \omega_{X^{\prime}}^{-1}\right)^{\vee}, X$ be the image of the natural map $X^{\prime} \rightarrow \mathbb{P}(V)$, and $\mathcal{L}=\mathcal{O}_{X}(1)$. Let $G$ be a connected algebraic group acting on $X$.

Theorem 1.1. The period integrals of the family $\pi: \mathcal{Y} \rightarrow B$ are solutions to

$$
\tau=\tau\left(G, X, \mathcal{L}, \beta_{0}\right),
$$

where $\beta_{0}$ is the Lie algebra homomorphism which vanishes on $\mathfrak{g}$ and $\beta_{0}(e)=1$.

This was proved in [16] for $X$ a partial flag variety, and in full generality in [17, where the result was also generalized to hyperplane sections of general type.

We note that when $X^{\prime}$ is a projective homogeneous manifold of a semisimple group $G$, in which case we have $X=X^{\prime}, \tau$ is amenable to explicit descriptions. For example, one description says that the tautological system can be generated by the vector fields corresponding to the linear $G$ action on $V^{\vee}$, and a twisted Euler vector field, together with a set of quadratic differential operators corresponding to the defining relations of $X$ in $\mathbb{P}(V)$ under the Plücker embedding. The case where $X$ is a Grassmannian has been worked out in detail [16. Furthermore, when the middle primitive cohomology $H^{n}(X)_{\text {prim }}=0$, it is also known that the system $\tau$ is complete, i.e. the solution sheaf coincides with the period sheaf [4]. 
We now return to a general tautological system $\tau$. Applying an argument of [12, we find that if $G$ acts on $X$ by finitely many orbits, and if the character D-module on $\hat{G}$

$$
\mathcal{L}_{\beta}:=D_{\hat{G}} / D_{\hat{G}}(\xi+\beta(\xi), \xi \in \hat{\mathfrak{g}})
$$

on $\hat{G}$ is regular singular, then $\tau$ is regular holonomic. See [16] Theorem 3.4(1). In this case, if $X=\sqcup_{l=1}^{r} X_{l}$ is the decomposition into $G$-orbits, then the singular locus of $\tau$ is contained in $\cup_{l=1}^{r} X_{l}^{\vee}$. Here $X_{l}^{\vee} \subset V^{\vee}$ is the conical variety whose projectivization $\mathbb{P}\left(X_{l}^{\vee}\right)$ is the projective dual to the Zariski closure of $X_{l}$ in $X$. From now on we assume that $G$ acts on $X$ by finitely many orbits, and $\mathcal{L}_{\beta}$ is regular singular. Note that the latter assumption is always satisfied when $G$ is reductive.

Let us now turn to the main problem studied in this paper. In the well-known applications of variation of Hodge structures in mirror symmetry, it is important to decide which solutions of our differential system come from period integrals. By Theorem 1.1, the period sheaf is a subsheaf of the solution sheaf of a tautological system. Thus an important problem is to decide when the two sheaves actually coincide, i.e. when $\tau$ is complete. If $\tau$ is not complete, how much larger is the solution sheaf relative to the period sheaf? From Hodge theory, we know that (see Proposition 6.3 [4) the rank of the period sheaf is given by the dimension of the middle vanishing cohomology of the smooth hypersurface $Y_{a}$. Therefore, to answer those questions, it is clearly desirable to know precisely the holonomic rank of $\tau$. For a brief overview of known results on these questions in a number of special cases, see Introduction in [4.

Conjecture 1.2. (Holonomic rank conjecture) Let $X$ be an $n$-dimensional projective homogeneous space of a semisimple group $G$. The solution $\operatorname{rank}$ of $\tau=\tau\left(G, X, \omega_{X}^{-1}, \beta_{0}\right)$ at the point $a \in V^{\vee}$ is given by $\operatorname{dim} H_{n}\left(X-Y_{a}\right)$.

In 4, the following is proved,

Theorem 1.3. Assume that the natural map

$$
\mathfrak{g} \otimes \Gamma\left(X, \omega_{X}^{-r}\right) \rightarrow \Gamma\left(X, T_{X} \otimes \omega_{X}^{-r}\right)
$$

is surjective for each $r \geq 0$. Then conjecture 1.2 holds.

In this paper, we prove this in full generality.

Theorem 1.4. Conjecture 1.2 holds.

This will be proved in $\$ 2$. There are at least two immediate applications of this result. First we can now compute the solution rank for $\tau$ for generic $a \in V^{\vee}$. 
Corollary 1.5. The solution rank of $\tau$ at a smooth hyperplane section a is

$$
\operatorname{dim} H^{n}(X)_{\text {prim }}+\operatorname{dim} H^{n-1}\left(Y_{a}\right)-\operatorname{dim} H^{n+1}(X)
$$

where the first term is the middle primitive cohomology of $X=G / P$ with $n=\operatorname{dim} X$.

The last two terms of the rank above can be computed readily in terms of the semisimple group $G$ and the parabolic subgroup $P$ by the Lefschetz hyperplane and the Riemann-Roch theorems. (See Example 2.4 [16]). A second application of Theorem 1.4 is to find certain exceptional points $a$ in $V^{\vee}$ where the solution sheaf of $\tau$ degenerates "maximally".

Definition 1.1. A nonzero section $a \in V^{\vee}=\Gamma\left(X, \omega_{X}^{-1}\right)$ is called a rank 1 point if the solution rank of $\tau$ at $a$ is 1 . In other words, $\operatorname{Hom}_{D_{V} \vee}\left(\tau, \mathcal{O}_{V^{\vee}, a}\right) \cong \mathbb{C}$.

Corollary 1.6. Any projective homogeneous variety admits a rank 1 point.

We will construct these rank 1 points in two explicit but different ways. The first, which works for $G=S L_{l}$, is a recursive procedure that produces such a rank 1 point by assembling rank 1 points from lower step flag varieties, starting from Grassmannians, and by repeatedly applying Theorem[1.4. The second way, which works for any semisimple group $G$, is by using a well-known $B$-invariant stratification of the flag variety $G / B$ to produce an open stratum in $X=G / P$ with a one dimensional middle degree cohomology. The complement of this stratum is an anticanonical divisor, hence a rank 1 point of $X$ by Theorem 1.4 .

The geometric formula in Conjecture 1.2 appears to go well beyond the context of homogeneous spaces. Theorem 1.4 can be seen as a special case of the following much more general theorem. Consider a smooth projective $G$-variety $X$ with $\mathcal{L}=\omega_{X}^{-1}$ very ample. Set $\tau=\tau(G, X, \mathcal{L}, \beta)$ and $V^{\vee}=\Gamma(X, \mathcal{L})$. We introduce some more notations. Let $\mathbb{L}^{\vee}$ be the total space of $\mathcal{L}$ and $\mathbb{L}^{\vee}$ be the complement of the zero section. Let

$$
e v: V^{\vee} \times X \rightarrow \mathbb{L}^{\vee}, \quad(a, x) \mapsto a(x)
$$

be the evaluation map, and $\mathbb{L}^{\perp}:=\operatorname{ker}(e v)$. Finally let

$$
\pi^{\vee}: U:=V^{\vee} \times X-\mathbb{L}^{\perp} \rightarrow V^{\vee}
$$

Note that this is the complement of the universal family of hyperplane sections $\mathbb{L}^{\perp} \rightarrow$ $V^{\vee},(a, x) \mapsto a$. Put

$$
D_{X, \beta}=\left(D_{X} \otimes k_{\beta}\right) \otimes_{U \hat{\mathfrak{g}}} k,
$$

where $k_{\beta}$ is the 1 -dimensional $\hat{\mathfrak{g}}$-module given by the character $\beta$ (see $\mathrm{A}$ for the notations). We now state the main results of this paper.

Theorem 1.7. For $\beta(e)=1$, there is a canonical isomorphism

$$
\left.\tau \simeq H^{0} \pi_{+}^{\vee}\left(\mathcal{O}_{V} \vee \otimes D_{X, \beta}\right)\right|_{U}
$$


Corollary 1.8. Suppose $G$ acts on $X$ by finitely many orbits, and $k=\mathbb{C}$. Then the solution rank of $\tau$ at $a \in V^{\vee}$ is given by $\operatorname{dim} H_{c}^{n}\left(U_{a},\left.\operatorname{Sol}\left(D_{X, \beta}\right)\right|_{U_{a}}\right)$, where $U_{a}=X-Y_{a}$.

More generally, we have

Theorem 1.9. For $\beta(e) \notin \mathbb{Z}_{\leq 0}$, there is a canonical isomorphism

$$
\tau \simeq H^{0} \pi_{+}^{\vee} e v^{!}\left(D_{\mathbb{L}^{\vee}, \beta}\right)[1-r] .
$$

In addition to proving Conjecture 1.2 as a special case, Theorem 1.7 can also be used to derive the well-known formula for the solution rank of a GKZ system [7] at generic point $a$. But since Corollary 1.8 holds for arbitrary $a \in V^{\vee}$, it holds in particular for $a$ corresponding the union of all $T$-invariant divisors in $X$ (which is anticanonical). In this case, Theorem 1.7 implies that $a$ is a rank 1 point - a result of [9] based on Gröbner basis theory but motivated by applications to mirror symmetry. Thus Theorem 1.7 and Corollary 1.8 interpolate a result of [7] and [9] by unifying the rank formula at generic point and at those exceptional rank 1 points, and at the same time, generalize them to an arbitrary $G$-variety.

We now outline the paper. In $₫ 2$ we prove Theorem 1.7 and a number of its consequences, including Theorem 1.4. We also describe explicitly the "cycle-to-period" map $H_{n}\left(X-Y_{a}\right) \rightarrow$ $\operatorname{Hom}_{D_{V} \vee}\left(\tau, \mathcal{O}_{V^{\vee}, a}\right)$ as a result of Theorem 1.7 and use it to answer a question recently communicated to us by S. Bloch. While $\$ 2$ deals only with the case $\beta(e)=1$, we remove this assumption in $\S \$ 35$. In $\$ 3$ we study the !-fibers of $\tau$, and describe some vanishing results at the special point $a=0$. We describe the geometric set up in $\$ 4$ for proving Theorem [1.9. The key step of the proof, involving an exact sequence for $\tau$, is done in $\$ 5$. Finally, we apply our results to construct rank 1 points for partial flag varieties in the case $G=S L_{l}$ in $\S \$ 7,8$ and for general semisimple groups in $\$ 6$. The appendix $\$ \mathrm{~A}$ collects some standard facts on D-modules.

Acknowledgements. S. Bloch has independently noticed the essential role of the RiemannHilbert correspondence in connecting the de Rham cohomology and solution sheaf of a tautological system. We thank him for kindly sharing his observation with us. A.H. would like to thank S.-T. Yau for advice and continuing support, especially for providing valuable resources to facilitate his research. B.H.L. is partially supported by NSF FRG grant DMS 1159049. X. Z. is supported by NSF grant DMS-1313894.

\section{CY hyPERPlane SECTIONS}

We begin with Theorem 1.7: $X$ is a $G$-variety with $\mathcal{L}=\omega_{X}^{-1}$ very ample, and $\beta(e)=1$. This is in fact a special case of the more general Theorem 1.9 and therefore can be also 
obtained by the methods introduced in later sections. However, we decide to deal with this case first for several reasons. On the one hand, the proof given here is different from the later method and is more direct. On the other hand, Theorem 1.7 already contains a subcase when $\left.\beta\right|_{\mathfrak{g}}=0$, i.e. $\beta=\beta_{0}$, which is important to mirror symmetry.

Let $n=\operatorname{dim} X$. Let $U=V^{\vee} \times X-V(f)$, where $V(f)=\mathbb{L}^{\perp}$ is the universal hyperplane section, so that $U_{a}=X-V\left(f_{a}\right)$ where $V\left(f_{a}\right)=Y_{a}$, the zero locus of the section $f_{a} \equiv a \in V^{\vee}$. Let $\pi^{\vee}: U \rightarrow V^{\vee}$ denote the projection. The restriction of $\beta$ to $\mathfrak{g}$ is still denoted by $\beta$ when no confusion arises. Put $D_{X, \beta}=\left(D_{X} \otimes k_{\beta}\right) \otimes_{U \mathfrak{g}} k$. Note that if $G$ acts on $X$ by finitely many orbits, then $D_{X, \beta}$ is $(G, \beta)$-equivariant holonomic D-module on $X$, and

$$
\mathcal{N}:=\left.\left(\mathcal{O}_{V \vee} \otimes D_{X, \beta}\right)\right|_{U}
$$

is a holonomic D-module on $U$.

Theorem 2.1. There is a canonical isomorphism $\tau \simeq H^{0} \pi_{+}^{\vee} \mathcal{N}$.

Corollary 2.2. If $\left.\beta\right|_{\mathfrak{g}}=0$. There is a canonical surjective map

$$
\tau \rightarrow H^{0} \pi_{+}^{\vee} \mathcal{O}_{U}
$$

Proof. Note that there is always a surjective map $D_{X, 0}=D_{X} / D_{X} \mathfrak{g} \rightarrow D_{X} / D_{X} T_{X}=\mathcal{O}_{X}$. The corollary follows.

Corollary 2.3. If $\left.\beta\right|_{\mathfrak{g}}=0$ and $X$ is a homogeneous $G$-variety, then $\tau \simeq H^{0} \pi_{+}^{\vee} \mathcal{O}_{U}$.

Proof. If $X$ is homogeneous, $\mathfrak{g} \otimes \mathcal{O}_{X} \rightarrow T_{X}$ is surjective. Therefore $D_{X, 0}=D_{X} / D_{X} T_{X}=$ $\mathcal{O}_{X}$

Note that this corollary implies that the tautological system in this case, which is a priori defined as a D-module by generators and relations, is of geometric origin, i.e. itself is a Gauss-Mannin connection.

We turn to the solution sheaf of $\tau$ via the Riemann-Hilbert correspondence. Assume $G$ acts on $X$ by finitely many orbits. Let us write $\mathcal{F}=\operatorname{Sol}\left(D_{X, \beta}\right)$. This is a perverse sheaf on $X$. In the case $X$ is a homogenous $G$-variety and $\left.\beta\right|_{\mathfrak{g}}=0$, then $\mathcal{F}=\mathbb{C}[n]$.

Corollary 2.4. Let $k=\mathbb{C}$ and $a \in V^{\vee}$. Then the solution rank of $\tau$ at a is given by $\operatorname{dim} H_{c}^{n}\left(U_{a},\left.\mathcal{F}\right|_{U_{a}}\right)$. 
Proof. To simplify the notation, we assume that $X$ is homogeneous and $\left.\beta\right|_{\mathfrak{g}}=0$. According to the Riemann-Hilbert correspondence, $\operatorname{Sol}(\tau)={ }^{p} R^{0} \pi_{!}^{\vee} \mathbb{C}_{U}[n+r]$, where ${ }^{p} R^{0} \pi_{!}^{\vee}$ denotes the 0th perverse cohomology of $\pi_{!}^{\vee}$. Then the non-derived solution sheaf $\operatorname{Hom}_{D_{V} \vee}\left(\tau, \mathcal{O}_{V^{\vee}}\right)$ is $H^{-r}\left({ }^{p} R^{0} \pi_{!}^{\vee} \mathbb{C}[n+r]\right)$, the $(-r)$ th (standard) sheaf cohomology of ${ }^{p} R^{0} \pi_{!}^{\vee} \mathbb{C}[n+r]$. However, as $R \pi_{!}^{\vee} \mathbb{C}[n+r]$ lives in positive perverse degrees, $H^{-r}\left({ }^{p} R^{0} \pi_{!}^{\vee} \mathbb{C}[n+r]\right)=H^{-r} R \pi_{!}^{\vee} \mathbb{C}[n+r]=$ $R^{n} \pi_{!}^{\vee} \mathbb{C}_{U}$. The claim follows.

Corollary 2.5. Conjecture 1.2 holds.

Now we prove Theorem 2.1. We will assume $\left.\beta\right|_{\mathfrak{g}}=0$ to simplify notations.

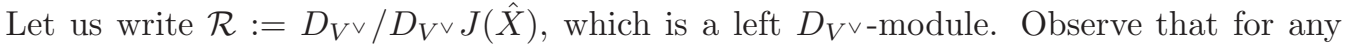
$\xi \in \hat{\mathfrak{g}}$,

$$
D_{V} I(\hat{X}) Z^{\vee}(\xi) \subset D_{V} I(\hat{X})
$$

and therefore

$$
D_{V \vee} J(\hat{X}) Z(\xi) \subset D_{V \vee} J(\hat{X}) .
$$

Therefore, $D_{V^{\vee}} J(\hat{X})$ can be regarded as a right $\hat{\mathfrak{g}}$-module, on which $\xi \in \hat{\mathfrak{g}}$ acts via the right multiplication by $Z(\xi)$. Accordingly, $\mathcal{R}$ is also a right $\hat{\mathfrak{g}}$-module. In addition, by definition we have

$$
\tau=\left(\mathcal{R} \otimes k_{\beta}\right) \otimes_{\hat{\mathfrak{g}}} k,
$$

where $k_{\beta}$ is the 1 -dimensional representation of $\hat{\mathfrak{g}}$ defined by $\beta$.

Let $\left\{a_{i}\right\}$ be a basis of $V$ and $\left\{a_{i}^{*}\right\}$ the dual basis. Observe that as $\mathcal{O}_{V^{\vee} \text {-modules, one can }}$ write

$$
\mathcal{R} \simeq \mathcal{O}_{V^{\vee}} \otimes S,
$$

where $S=k\left[\partial_{a_{i}}\right] / J(\hat{X}) \simeq \mathcal{O}_{V} / I(\hat{X})$ is identified with the homogeneous coordinate ring of $\hat{X}$, and $\mathcal{O}_{V^{\vee}}$ acts on the first factoly. If we convert the right action of $\hat{\mathfrak{g}}$ on $\mathcal{R}$ described above to a left action $\alpha$, then $\alpha$ will be the sum of the following two actions: the first is the action of $\hat{\mathfrak{g}}$ on the second factor through $Z: \hat{\mathfrak{g}} \rightarrow \operatorname{End} V \rightarrow \operatorname{End} S$, which is denoted by $\alpha_{1}$; to describe the second action $\alpha_{2}$, observe that the natural multiplication map

$$
\left(V \otimes V^{\vee}\right) \otimes\left(\mathcal{O}_{V^{\vee}} \otimes S\right) \rightarrow\left(\mathcal{O}_{V^{\vee}} \otimes S\right),
$$

induces $V \otimes V^{\vee} \rightarrow \operatorname{End} \mathcal{R}$ and $\alpha_{2}$ is via $Z: \hat{\mathfrak{g}} \rightarrow V \otimes V^{\vee} \rightarrow \operatorname{End}(\mathcal{R})$. Explicitly, if we write $a \otimes b \in \mathcal{O}_{V^{\vee}} \otimes S$, then

$$
\alpha_{1}(\xi)(a \otimes b)=a \otimes Z(\xi)(b) .
$$

Let's write $Z(\xi)=\sum_{i j} \xi_{i j} a_{i} \otimes a_{j}^{*}$. Then

$$
\alpha_{2}(\xi)(a \otimes b)=\sum_{i j} \xi_{i j} a a_{i} \otimes b a_{j}^{*}
$$

\footnotetext{
${ }^{1}$ The $D_{V} \vee$-module structure on $\mathcal{R}$ is given as follows: $\partial_{a_{i}}$ acts on $\mathcal{R}$ as $\partial_{a_{i}} \otimes 1+1 \otimes a_{i}^{*}$.
} 
Let $f=\sum a_{i} \otimes a_{i}^{*} \in \mathcal{R}$, which can be regarded as the universal section of the line bundle $\mathcal{O}_{V} \vee \otimes \mathcal{L}$ over $V^{\vee} \times X$. Recall that $U=V^{\vee} \times X-\mathbb{L}^{\perp}$. Then

$$
\mathcal{O}_{U}=\left(\mathcal{O}_{V^{\vee}} \otimes S(\hat{X})\right)_{(f)}
$$

is the homogeneous localization of $\mathcal{R}$ with respect to $f$, where the degree of $a \otimes b \in \mathcal{O}_{V} \vee \otimes$ $S(\hat{X})$ is the degree of $b$ in the graded ring $S(\hat{X})$. As $\mathcal{L}^{-1}=\omega_{X}, f^{-1}$ can be regarded as a rational section of $\mathcal{O}_{V} \vee \otimes \omega_{X}$, regular on $U$. Then $\mathcal{O}_{U} f^{-1}$ can be identified with the regular sections of $\mathcal{O}_{V} \vee \otimes \omega_{X}$ over $U$. In other words,

$$
\mathcal{O}_{U} f^{-1} \simeq \omega_{U / V^{\vee}}=\left.\left(\mathcal{O}_{V^{\vee}} \otimes \omega_{X}\right)\right|_{U} .
$$

Therefore, it is equipped with a $\left.\left(D_{V^{\vee}} \otimes D_{X}^{o p}\right)\right|_{U}$ module structure (see [5, VI, §3] for the definition of right $D_{X}$ module structure on $\left.\omega_{X}\right)$. As $\mathfrak{g}$ maps to the vector fields on $X, \mathcal{O}_{U} f^{-1}$ is a $D_{V} \vee \times \mathfrak{g}$-module. We will describe this structure more explicitly. First, we describe the

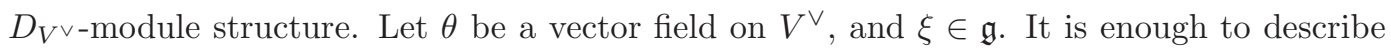
$\theta\left(f^{-1}\right)$ and $\left(f^{-1}\right) \xi$. Let us write $Z(\xi)=\sum \xi_{i j} a_{i} \otimes a_{j}^{*}$ as before.

Lemma 2.6. We have

$$
\theta\left(f^{-1}\right)=\frac{\sum_{i} \theta\left(a_{i}\right) \otimes a_{i}^{*}}{f^{2}} \in \mathcal{O}_{U} f^{-1}
$$

and

$$
\left(f^{-1}\right) \xi=\frac{\sum \xi_{i j} a_{i} \otimes a_{j}^{*}}{f^{2}} \in \mathcal{O}_{U} f^{-1}
$$

Proof. Let $v \in V^{\vee}$, regarded as a section of $\mathcal{L}$. Then $v^{-1}$ is a rational section of $\omega_{X}$, and $\omega=1 \otimes v^{-1}$ is a rational section of $\mathcal{O}_{V} \vee \otimes \omega_{X}$, obtained by pullback of a rational section of $\omega_{X}$. Note that $g=(1 \otimes v) / f \in \mathcal{O}_{U}$, and we can write $f^{-1}=g\left(1 \otimes v^{-1}\right)$. By definition, for a vector field $\theta$ on $V^{\vee}, \theta(\omega)=0$, and for $\xi \in \mathfrak{g}, \xi(\omega)=-1 \otimes \operatorname{Lie}_{\xi} v^{-1}$, where $\operatorname{Lie}_{\xi}: \omega_{X} \rightarrow \omega_{X}$ is the Lie derivative (see [5, VI, §3] for the definition of right D-module structures on $\omega_{X}$ ). Therefore

$$
\theta\left(f^{-1}\right)=\theta(g) \omega, \quad\left(f^{-1}\right) \xi=-\xi(g) \omega-g\left(1 \otimes \operatorname{Lie}_{\xi} v^{-1}\right) .
$$

Note that

$$
\theta(g)=\theta\left(\frac{1}{\sum a_{i} \otimes \frac{a_{i}^{*}}{v}}\right)=\frac{\sum \theta\left(a_{i}\right) \otimes \frac{a_{i}^{*}}{v}}{\left(\sum a_{i} \otimes \frac{a_{i}^{*}}{v}\right)^{2}}=g^{2} \sum \theta\left(a_{i}\right) \otimes \frac{a_{i}^{*}}{v}=g \frac{\sum \theta\left(a_{i}\right) \otimes a_{i}^{*}}{f} .
$$

Therefore, the first equation holds. On the other hand

$$
\xi(g)=\xi\left(\frac{1 \otimes v}{f}\right)=\frac{1 \otimes Z(\xi)(v)}{f}-\frac{(1 \otimes v) \sum \xi_{i j} a_{i} \otimes a_{j}^{*}}{f^{2}} .
$$

To prove the second, we need to understand $\operatorname{Lie}_{\xi} v^{-1}$. We consider the general siuation. Let $X$ be a Fano projective variety. Assume that $\mathcal{L}=\omega_{X}^{-1}$ is very ample, and $X \rightarrow \mathbb{P}(V)$ be the closed embedding where $V=\Gamma(X, \mathcal{L})^{\vee}$. Then $\mathfrak{g}=\Gamma\left(X, T_{X}\right)$ is a Lie algebra and $\mathcal{L}$ is naturally $\mathfrak{g}$-linearized. Therefore, $V$ is a natural $\mathfrak{g}$-module with action $Z: \mathfrak{g} \rightarrow \operatorname{End}(V)$. As $Z(\xi)=\sum \xi_{i j} a_{i} \otimes a_{j}^{*}$, we have $Z(\xi)(v)=\sum \xi_{i j} a_{i}(v) a_{j}^{*}$ for $v \in V^{\vee}$. On the other hand, recall that $\mathfrak{g}$ acts on $\omega_{X}$ by Lie derivatives. Note that for $v \in V^{\vee}, v^{-1}$ can be regarded as a rational section of $\omega_{X}$. 
Lemma 2.7. Let $\xi \in \mathfrak{g}, 0 \neq v \in V^{\vee}$ and write $Z(\xi)=\sum \xi_{i j} a_{i} \otimes a_{j}^{*}$. Then

$$
\operatorname{Lie}_{\xi} v^{-1}=-\frac{Z(\xi)(v)}{v} v^{-1} \text {. }
$$

Now Lemma 2.6 follows.

Note that explicitly, the $D_{V^{\vee}} \times \mathfrak{g}$-module structure on $\mathcal{O}_{U} f^{-1}$ can be described as follows. Let $\theta=\partial_{a_{i}}$ be a vector field on $V^{\vee}$ and $\xi=\sum \xi_{i j} a_{i} \otimes a_{j}^{*} \in \mathfrak{g}, m=\frac{1}{f^{k+1}}(a \otimes b) \in \mathcal{O}_{U} f^{-1}$, where $a \in \mathcal{O}_{V \vee}$ and $b \in S$ is homogeneous of degree $k$, then

$$
\begin{gathered}
\partial_{a_{i}}(m)=\frac{\partial_{a_{i}}(a) \otimes b}{f^{k+1}}+(-1)^{k+1}(k+1) \frac{a \otimes b a_{i}^{*}}{f^{k+2}}, \\
(m) \xi=-\frac{1}{f^{k+1}}(a \otimes Z(\xi)(b))+\frac{k+1}{f^{k+2}}\left(\sum \xi_{i j} a a_{i} \otimes b a_{j}^{*}\right) .
\end{gathered}
$$

We extend this to a $\hat{\mathfrak{g}}$-module by requiring that $e$ acts by zero on $\mathcal{O}_{U} f^{-1}$.

Now, we have the following technical lemma. Recall that $\beta(e)=1$.

Lemma 2.8. The map $\phi: \mathcal{R} \otimes \beta \rightarrow \mathcal{O}_{U} f^{-1}$ given by

$$
\phi(a \otimes b)=\frac{(-1)^{k} k !}{f^{k+1}} a \otimes b
$$

is a $D_{V^{\vee}} \times \hat{\mathfrak{g}}$-module homomorphism. In addition, it induces an isomorphism

$$
\tau=(\mathcal{R} \otimes \beta) \otimes_{\hat{\mathfrak{g}}} k \simeq\left(\mathcal{O}_{U} f^{-1}\right) \otimes_{\hat{\mathfrak{g}}} k=\left(\mathcal{O}_{U} f^{-1}\right) \otimes_{\mathfrak{g}} k .
$$

Proof. A direct calculation shows that $\phi$ is a $D_{V^{\vee}} \times \hat{\mathfrak{g}}$-module homomorphism. Namely, we know that $\partial_{a_{i}}$ acts on $\mathcal{R}$ by $\partial_{a_{i}} \otimes 1+1 \otimes a_{i}^{*}$. Therefore,

$\phi\left(\partial_{a_{i}}(a \otimes b)\right)=\phi\left(\partial_{a_{i}}(a) \otimes b+a \otimes b a_{i}^{*}\right)=\frac{(-1)^{k} k !}{f^{k+1}}\left(\partial_{a_{i}}(a) \otimes b\right)+\frac{(-1)^{k+1}(k+1) !}{f^{k+2}}\left(a \otimes b a_{i}^{*}\right)$,

which is the same as $\partial_{a_{i}} \phi(a \otimes b)$. The $\mathfrak{g}$-equivariance can be checked similarly.

Clearly $\phi$ is surjective, with the kernel spanned by $(k+1) a \otimes b+f(a \otimes b)$ for $b$ homogeneous of degree $k$. But $(a \otimes b) \alpha(e)=(k+1) a \otimes b+f(a \otimes b)$. The lemma is proved.

To apply this lemma, recall the definition of $\pi_{+}^{\vee}$ for $\pi^{\vee}: U \rightarrow V^{\vee}$ a smooth morphism of algebraic varieties. As $\pi^{\vee}$ is an affine morphism,

$$
\pi_{+}^{\vee} \mathcal{N}=\Omega_{U / V^{\vee}}^{\bullet} \otimes \mathcal{N}[\operatorname{dim} X] .
$$

In particular,

$$
H^{0} \pi_{+}^{\vee} \mathcal{N}=\operatorname{coker}\left(\left.\left.\left(\mathcal{O}_{V \vee} \otimes \Omega_{X}^{\operatorname{dim} X-1} \otimes D_{X} \otimes_{U \mathfrak{g}} k\right)\right|_{U} \rightarrow\left(\mathcal{O}_{V^{\vee}} \otimes \omega_{X} \otimes D_{X} \otimes_{U \mathfrak{g}} k\right)\right|_{U}\right) .
$$

As $\operatorname{coker}\left(\Omega_{X}^{\operatorname{dim} X-1} \otimes D_{X} \rightarrow \omega_{X} \otimes D_{X}\right)=\omega_{X}$ as right $D_{X}$-modules, $H^{0} \pi_{+}^{\vee} \mathcal{N}$ is exactly $\left.\left(\mathcal{O}_{V} \vee \otimes \omega_{X}\right)\right|_{U} \otimes_{U \mathfrak{g}} k \simeq \tau$. This completes the proof of Theorem 2.1] 
We continue to let $X$ be a general smooth projective $G$-variety, and let $\beta(e)=1$ and $\left.\beta\right|_{\mathfrak{g}}=0$, and consider some consequences of Theorem 2.1. By taking the solution sheaves on both sides in Corollary 2.2, we get an injective map

$$
H_{n}\left(X-V\left(f_{a}\right)\right) \rightarrow \operatorname{Hom}\left(\tau, \mathcal{O}_{V^{\vee}, a}\right) .
$$

This gives an explicit lower bound for the solution $\operatorname{rank}$ of $\tau$ at any point $a$. It is interesting to compare this with the "cycle-to-period" map constructed in [17] when $k=\mathbb{C}$. The latter can be described in differential geometric terms as follows. For $\mathcal{L}=\omega_{X}^{-1}$, the $D_{V^{\vee}} \times \mathfrak{g}$-module isomorphism (2.1) is given, at each point $a$, by

$$
\frac{1}{f_{a}} \mapsto \frac{\Omega}{f_{a}}
$$

where $\Omega$ is the canonical $G$-invariant $\mathbb{G}_{m}$-horizontal form of degree $\operatorname{dim} X$ on $\mathbb{L}^{\vee}-\{0\}$ $([17, \S 4])$. Here $\frac{\Omega}{f_{a}}$ is a $\mathbb{G}_{m}$-invariant meromorphic form on the principal $\mathbb{G}_{m}$-bundle $\pi^{\vee}$ : $\left(\mathbb{L}^{\vee}-\{0\}\right) \rightarrow X$, and thus can be viewed as a meromorphic form on the base $X$ with pole along $V\left(f_{a}\right)$. It follows that

$$
\tau=\mathcal{O}_{U} f^{-1} \otimes_{\mathfrak{g}} k \simeq \omega_{U / V^{\vee}} \otimes_{\mathfrak{g}} k, \quad 1 \mapsto \frac{\Omega}{f_{a}}
$$

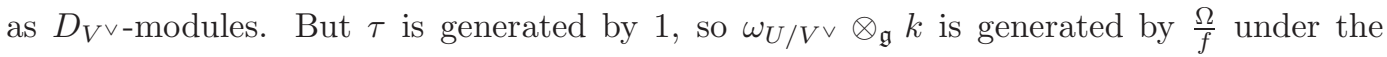
Gauss-Manin connection.

Corollary 2.9. The cycle-to-period map $H_{n}\left(X-V\left(f_{a}\right)\right) \rightarrow \operatorname{Hom}\left(\tau, \mathcal{O}_{V^{\vee}, a}\right)$,

$$
\gamma \mapsto\left\langle\gamma, \frac{\Omega}{f_{a}}\right\rangle=\int_{\gamma} \frac{\Omega}{f_{a}},
$$

is injective.

Proof. For $\gamma \in \operatorname{Hom}\left(H^{0} \pi_{+}^{\vee} \mathcal{O}_{U}, \mathcal{O}_{V^{\vee}, a}\right)=H_{n}\left(X-V\left(f_{a}\right)\right)$, its image is a $D_{V^{\vee}}$-linear function $\tau \simeq \omega_{U / V^{\vee}} \otimes_{\mathfrak{g}} k \rightarrow \mathcal{O}_{V^{\vee}, a}$. Since $\tau_{a}$ is generated by $\frac{\Omega}{f_{a}}$, if $\left\langle\gamma, \frac{\Omega}{f_{a}}\right\rangle=0$ then $\left\langle\gamma, \tau_{a}\right\rangle=0$, hence $\gamma=0$

We also have the following interesting topological consequence, which answers a question S. Bloch communicated to us.

Corollary 2.10. Let $a \in V^{\vee}$. Then for $a^{\prime}$ close to a, the map $H_{n}\left(X-V\left(f_{a}\right)\right) \rightarrow H_{n}(X-$ $\left.V\left(f_{a^{\prime}}\right)\right)$ induced by parallel transport is injective.

Proof. Since any analytic solution to $\tau$ at $a$ extends to some neighborhood of $a$, the map $\operatorname{Hom}\left(\tau, \mathcal{O}_{V^{\vee}, a}\right) \rightarrow \operatorname{Hom}\left(\tau, \mathcal{O}_{V^{\vee}, a^{\prime}}\right)$ given by analytic continuation is injective. Now the claim follows from Corollary 2.9 .

Remark 2.1. The two preceding corollaries rely only on the fact that $\omega_{U / V^{\vee}} \otimes_{\mathfrak{g}} k$ is generated

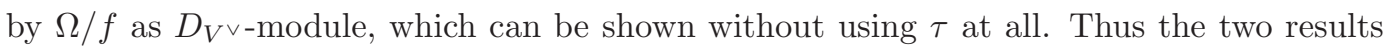
are general facts about the D-module $H \pi_{+}^{\vee} \mathcal{N}$, as apposed to $\tau$ specifically. 


\section{3. !-FIBERS OF $\tau$}

In the following three sections, we consider $\tau$ when $\beta(e)$ is not necessarily 1 . Here we will give a formula of the !-fibers of $\tau$ at $a \in V^{\vee}$. For $a \in V^{\vee}$, let $i_{a}:\{a\} \rightarrow V^{\vee}$ be the inclusion and for simplicity, let us write

$$
\tau_{a}^{!}=i_{a}^{!} \tau
$$

This is a complex of vector spaces and our goal is to give an expression of this complex.

Recall that

$$
\tau=(\mathcal{R} \otimes \beta) \otimes_{\hat{\mathfrak{g}}} k .
$$

As a result, we have

$$
\tau_{a}^{!}=k_{a} \otimes_{\mathcal{O}_{V^{\vee}}}^{L}\left((\mathcal{R} \otimes \beta) \otimes_{\hat{\mathfrak{g}}} k\right)[-\operatorname{dim} V],
$$

where $k_{a}=\mathcal{O}_{V^{\vee}} / \mathfrak{m}_{a}$ is the residual field at $a$, and $\mathfrak{m}_{a}$ is the maximal ideal of $\mathcal{O}_{V^{\vee}}$ corresponding to $a$.

The advantage of this expression of $\tau_{a}^{!}$is that we can first calculate $k_{a} \otimes_{\mathcal{O}_{V^{\vee}}}^{L} \mathcal{R}$ as a (complex of) right $\hat{\mathfrak{g}}$-modules, and then taking the Lie algebra coinvariants. Namely, we have the Koszul resolution of $k_{a}$, which gives the complex that calculates $\tau_{a}^{!}$

$$
\tau_{a}^{!}=\left(\bigwedge V \otimes \mathcal{O}_{V^{\vee}} \otimes S\right) \otimes_{\hat{\mathfrak{g}}}(-\beta) .
$$

where $V \otimes \mathcal{O}_{V^{\vee}} \rightarrow \mathcal{O}_{V^{\vee}}$ is given by $v \otimes 1 \mapsto v-v(a)$. In general, this complex is difficult to compute. However, when $a=0$, this is more tractable.

In fact, at $a=0$, for the degree $r$-term, we have

$$
H^{r} \tau_{0}^{!} \simeq H_{0}(\hat{\mathfrak{g}}, S \otimes \beta),
$$

where $\mathfrak{g}$ acts on $S$ via $Z: \hat{\mathfrak{g}} \rightarrow \operatorname{End} V \rightarrow \operatorname{End} S, Z(\xi)=\sum_{i j} \xi_{i j} a_{i} \otimes a_{j}^{*}$.

Corollary 3.1. Assume that $\beta(e) \notin \mathbb{Z}_{\leq 0}$. Then $H^{r} \tau_{0}^{!}=0$.

Proof. The homothethy $\mathbb{G}_{m}$ acts on $S$ by nonnegative weights. Therefore, if $\beta(e) \notin \mathbb{Z}_{\leq 0}$, the coinvariant of $S \otimes \beta$ with respect to this $\mathbb{G}_{m}$ is zero.

Remark 3.1. For a general point $a \in V^{\vee}$ we can still express $H^{r} \tau_{a}^{!} \simeq H_{0}(\hat{\mathfrak{g}}, S \otimes \beta)$. But if $a \neq 0$, the action of $\hat{\mathfrak{g}}$ on $S$ will be the sum of two actions (induced by the actions $\alpha_{1}$ and $\alpha_{2}$ of $\mathfrak{g}$ on $\mathcal{O}_{V \vee} \otimes S$, as described $₫ 2$ right after Corollary 2.4). Concretely, the first action is via $Z$, and the second is via the $\xi(b)=\sum \xi_{i j} a_{i}(a) b a_{j}^{*}$ for $b \in S$. Therefore, unless $a=0$, $S$ is not a finite dimensional $\hat{\mathfrak{g}}$-module and is difficult to compute. 
From now on, we assume that $\beta(e) \notin \mathbb{Z}_{\leq 0}$.

Let us calculate $H^{r-1} \tau_{0}^{!}$. We have

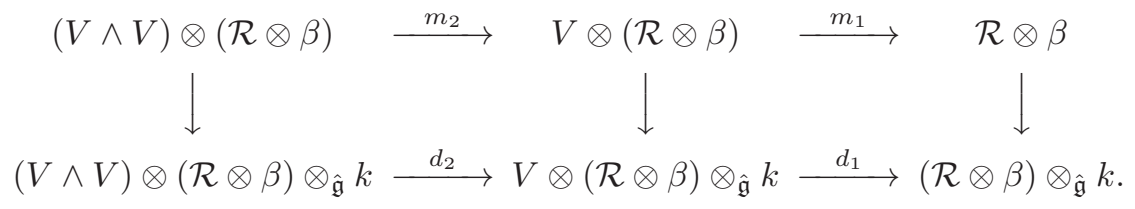

Then

$$
H^{r-1} \tau_{0}^{!}=m_{1}^{-1}((\mathcal{R} \otimes \beta) \hat{\mathfrak{g}}) /\left(\operatorname{Im} m_{2}+V \otimes(\mathcal{R} \otimes \beta) \hat{\mathfrak{g}}\right)
$$

As the Koszul complex is acyclic away from degree zero, we can rewrite the above as

$$
H^{r-1} \tau_{0}^{!}=(\mathcal{R} \otimes \beta) \hat{\mathfrak{g}} \cap \operatorname{Im} m_{1} /\left(\operatorname{Im} m_{1}\right) \hat{\mathfrak{g}} .
$$

Consider

$0 \rightarrow(\mathcal{R} \otimes \beta) \hat{\mathfrak{g}} \cap \operatorname{Im} m_{1} /\left(\operatorname{Im} m_{1}\right) \hat{\mathfrak{g}} \rightarrow(\mathcal{R} \otimes \beta) \hat{\mathfrak{g}} /\left(\operatorname{Im} m_{1}\right) \hat{\mathfrak{g}} \rightarrow(\mathcal{R} \otimes \beta) \hat{\mathfrak{g}} /(\mathcal{R} \otimes \beta) \hat{\mathfrak{g}} \cap \operatorname{Im} m_{1} \rightarrow 0$

Note that $0=H^{r} \tau_{0}^{!}$implies that $(\mathcal{R} \otimes \beta) \hat{\mathfrak{g}}+\operatorname{Im} m_{1}=\mathcal{R} \otimes \beta$. Therefore,

$$
(\mathcal{R} \otimes \beta) \hat{\mathfrak{g}} /(\mathcal{R} \otimes \beta) \hat{\mathfrak{g}} \cap \operatorname{Im} m_{1}=\mathcal{R} \otimes \beta / \operatorname{Im} m_{1} .
$$

We therefore can write

$$
H^{r-1} \tau_{0}^{!}=\operatorname{ker}\left((\mathcal{R} \otimes \beta) \hat{\mathfrak{g}} /\left(\operatorname{Im} m_{1}\right) \hat{\mathfrak{g}} \rightarrow \mathcal{R} \otimes \beta / \operatorname{Im} m_{1}\right) .
$$

Therefore, there is a surjective map

$$
H_{1}(\hat{\mathfrak{g}}, S \otimes \beta) \rightarrow H^{r-1} \tau_{0}^{!}
$$

where $\hat{\mathfrak{g}}$ acts on $S$ via $Z$. (So $S$ are direct sums of finite dimensional representations of $\hat{\mathfrak{g}}$.)

Lemma 3.2. For $\beta(e) \notin \mathbb{Z}_{\leq 0}$, we have $H_{1}(\hat{\mathfrak{g}}, S \otimes \beta)=0$.

Proof. Consider the $\hat{\mathfrak{g}}$ coinvariants functor as the composition of $\mathfrak{g}$ coinvariants functor, and the $\mathbb{C}$ coinvariants functor. The $E_{2}$ terms of the Grothendieck spectral sequence contributing to $H_{1}(\hat{\mathfrak{g}}, S \otimes \beta)$ are $H_{1}\left(\mathbb{C}, H_{0}(\mathfrak{g}, S \otimes \beta)\right)$ and $H_{0}\left(\mathbb{C}, H_{1}(\mathfrak{g}, S \otimes \beta)\right)$. As $S \otimes \beta$ breaks as direct sums according to weights as a $\mathfrak{g}$-module, and $\mathbb{C}$ acts on each given weight piece as the weight plus $\beta(e)$, it is clear that under the above assumption on $\beta(e)$, both $H_{1}\left(\mathbb{C}, H_{0}(\mathfrak{g}, S \otimes \beta)\right)$ and $H_{0}\left(\mathbb{C}, H_{1}(\mathfrak{g}, S \otimes \beta)\right)$ are zero. 


\section{The Geometry}

Let $X$ be a smooth projective variety and $\mathcal{L}$ a very ample line bundle which gives $X \rightarrow$ $\mathbb{P}(V)$, where $V^{\vee}=\Gamma(X, \mathcal{L})$. Let $\hat{\imath}: \hat{X} \rightarrow V$ be the closed embedding of the cone of $X$ into $V$. Let $\mathbb{L}$ be the totally space of $\mathcal{L}^{\vee}$. Then

$$
i_{\mathbb{L}}: \mathbb{L} \rightarrow X \times V
$$

is a rank one subbundle of the trivial vector bundle over $X$ with fiber $V$. The following diagram is commutative

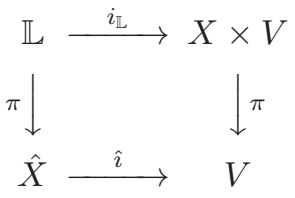

and the left vertical arrow realizes $\mathbb{L}$ as the blow-up of $\hat{X}$ at the origin. We denote the open immersion

$$
j_{\mathbb{L}}: \stackrel{\circ}{\mathbb{L}}=\mathbb{L}-X \rightarrow \mathbb{L},
$$

where $X$ is regarded as the zero section of $\mathbb{L}$.

Let $\mathbb{L}^{\vee}$ be the dual of $\mathbb{L}$, i.e., the total space of $\mathcal{L}$, and $j_{\mathbb{L}^{\vee}}: \mathbb{L}^{\vee} \rightarrow \mathbb{L}^{\vee}$ be the open subset away from the zero section. The the dual of $i_{\mathbb{L}}$ is the evaluation map

$$
e v: X \times V^{\vee} \rightarrow \mathbb{L}^{\vee}
$$

which sends $(x, a)$ to $a(x) \in \mathbb{L}^{\vee}$.

Let $i_{\mathbb{L}^{\perp}}: \mathbb{L}^{\perp} \rightarrow X \times V^{\vee}$ be the orthogonal complement of $\mathbb{L}$ in $X \times V^{\vee}$, i.e. the kernel of $e v$. The projection

$$
\mathbb{L}^{\perp} \stackrel{i_{\mathbb{L}}}{\rightarrow} X \times V^{\vee} \stackrel{\pi^{\vee}}{\rightarrow} V^{\vee}
$$

realizes $\mathbb{L}^{\perp}$ as the universal family of hyperplane sections of $X$. We still denote this projection by $\pi^{\vee}$. Let $j_{U}: U=X \times V^{\vee}-\mathbb{L}^{\perp} \rightarrow X \times V^{\vee}$ be the complement. For $a \in V^{\vee}$, the fiber $U_{a}$ of $U \rightarrow V^{\vee}$ over $a$ is $X-V\left(f_{a}\right)$, where $f_{a}$ is the section of $\mathcal{L}$ given by $a$ and $V\left(f_{a}\right)$ is its divisor. Note that the following diagram is Cartesian.

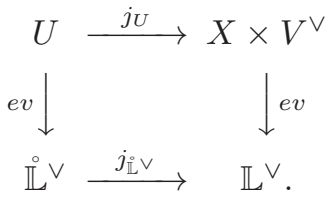




\section{An EXACT SEQUENCE FOR $\tau$}

We will complete the proof of Theorem 1.9 in this section.

Let $i_{0}:\{0\} \rightarrow V$ be the inclusion of the origin, and $j_{0}: \stackrel{\circ}{V} \rightarrow V$ be the open embedding of the complement. Let $\stackrel{\circ}{X}=\hat{X}-\{0\}$. The open inclusion $\stackrel{\circ}{X} \rightarrow \hat{X}$ is still denoted by $j_{0}$ and the closed inclusion $\stackrel{\circ}{X} \rightarrow \stackrel{\circ}{V}$ is denoted by $i$. By specializing (A.4), we have the following important sequence for $\hat{\tau}=\mathcal{F}$ our $(\tau)$

$$
0 \rightarrow i_{0,+} H^{-1} i_{0}^{+} \hat{\tau} \rightarrow H^{0} j_{0, !}\left(\left.\hat{\tau}\right|_{\text {V }}\right) \rightarrow \hat{\tau} \rightarrow i_{0,+} H^{0} i_{0}^{+} \hat{\tau} \rightarrow 0
$$

First we make a simplification of this sequence.

Lemma 5.1. For $\beta(e) \notin \mathbb{Z}_{\leq 0}, i_{0,+} H^{0} i_{0}^{+} \hat{\tau}=0$.

Proof. Assume that $H^{0} i_{0}^{+} \hat{\tau}=k^{\ell}$, so that $i_{0,+} H^{0} i_{0}^{+} \hat{\tau}=\delta_{0}^{\ell}$. I.e. there is a surjective map of D-modules $\hat{\tau} \rightarrow \delta_{0}^{\ell}$ on $V$. Taking the Fourier transform, we therefore have a surjective map $\tau \rightarrow \mathcal{O}_{V^{\vee}}^{\ell}$. Taking the right exact functor $H^{r} i_{0}^{!}$, i.e., the $r$ th cohomology of the !-fibers at $0 \in V^{\vee}$, we have a surjective map $H^{r} \tau_{0}^{!} \rightarrow k^{\ell}$. By Corollary 3.1 $\ell=0$.

As a result, under our assumption

$$
0 \rightarrow i_{0,+} H^{-1} i_{0}^{+} \hat{\tau} \rightarrow H^{0} j_{0, !}\left(\left.\hat{\tau}\right|_{\hat{V}}\right) \rightarrow \hat{\tau} \rightarrow 0 .
$$

Let $d=\operatorname{dim}_{k} H^{-1} i_{0}^{+} \hat{\tau}$. Then $i_{0,+} H^{-1} i_{0}^{+} \hat{\tau}=\delta_{0}^{d}$. Taking the Fourier transform of this sequence, we therefore obtain

$$
0 \rightarrow \mathcal{O}_{V^{\vee}}^{d} \rightarrow \mathcal{F} \text { our }\left(H^{0} j_{0, !}\left(\left.\hat{\tau}\right|_{V}\right)\right) \rightarrow \tau \rightarrow 0 .
$$

We next understand $\mathcal{F}$ our $\left(H^{0} j_{0, !}\left(\left.\hat{\tau}\right|_{\hat{V}}\right)\right)$. Clearly, $\hat{\tau}$ is set-theoretically supported on $\hat{X}$. The following lemma is clear.

Lemma 5.2. For $\mathcal{L}=\omega_{X}^{-1}$, we have $\left.\hat{\tau}\right|_{\dot{X}}=D_{\dot{X}, \beta^{\prime}}$, where $D_{\dot{X}, \beta^{\prime}}$ is the D-module on $\AA_{X}$ as introduced in Lemma A.6.

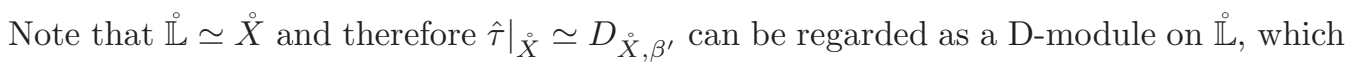
is naturally ( $\mathbb{G}_{m}, \beta^{\prime}(e)$ )-equivariant. Then

$$
H^{0} j_{0, !}\left(\left.\hat{\tau}\right|_{\dot{V}}\right)=H^{0} \pi_{!}\left(i_{\mathbb{L}, !} j_{\mathbb{L}, !} D_{\dot{X},-\beta^{\prime}}\right)
$$

According to Lemma A.14.

$$
\mathcal{F} \text { our }\left(H^{0} \pi_{!}\left(i_{\mathbb{L}, !} j_{\mathfrak{L}, !} D_{\dot{X},-\beta^{\prime}}\right)\right)=H^{0} \pi_{!}^{\vee} \mathcal{F}_{\text {our }}\left(i_{\mathbb{L}, !} j_{\mathfrak{L}, !} D_{\dot{X},-\beta^{\prime}}\right) .
$$


By (A.7),

$$
\mathcal{F}_{\text {our }_{X}}\left(i_{\mathbb{L}, !} j_{\mathbb{L}, !} D_{\hat{X},-\beta^{\prime}}\right)=e v^{!} \mathcal{F} \operatorname{our}_{X}\left(j_{\mathfrak{L}, !} D_{\dot{X},-\beta^{\prime}}\right)[1-r] .
$$

Finally,

Lemma 5.3. There is an isomorphism

$$
\operatorname{Four}_{X}\left(j_{\mathbb{L}, !} D_{\grave{X},-\beta^{\prime}}\right) \simeq j_{\mathscr{L},+} D_{\mathbb{L}^{\vee},-\beta} .
$$

Lemma 5.4. Assume that $\beta(e) \notin \mathbb{Z}_{\leq 0}$. We have $d=\operatorname{dim} H^{r-1} \tau_{0}^{!}=0$.

Proof. The second statement follows from 3.2 We need to establish the first equality. For simplicity, let us denote $\mathcal{N}:=e v^{!}\left(D_{\mathbb{L} v,-\beta}\right)[1-r]$. This is a plain D-module on $U$.

Taking $i_{0}^{!}$of (5.1), it is enough to show that

$$
H^{r} i_{0}^{!} H^{0} \pi_{+}^{\vee} \mathcal{N}=H^{r-1} i_{0}^{!} H^{0} \pi_{+}^{\vee} \mathcal{N}=0 .
$$

Consider the distinguished triangle

$$
i_{0}^{!} H^{\leq-1} \pi_{+}^{\vee} \mathcal{N} \rightarrow i_{0}^{!} \pi_{+}^{\vee} \mathcal{N} \rightarrow i_{0}^{!} H^{0} \pi_{+}^{\vee} \mathcal{N} \rightarrow .
$$

The long exact sequence associated to this triangle is

$$
H^{r-1} i_{0}^{!} \pi_{+}^{\vee} \mathcal{N} \rightarrow H^{r-1} i_{0}^{!} H^{0} \pi_{+}^{\vee} \mathcal{N} \rightarrow H^{r} i_{0}^{!} H^{\leq-1} \pi_{+}^{\vee} \mathcal{N} \rightarrow H^{r} i_{0}^{!} \pi_{+}^{\vee} \mathcal{N} \rightarrow H^{r} i_{0}^{!} H^{0} \pi_{+}^{\vee} \mathcal{N} \rightarrow 0 .
$$

Note that $U$ does not intersect with $X \times\{0\} \subset X \times V^{\vee}$. Therefore, $i_{0}^{!} \pi_{+}^{\vee} \mathcal{N}=0$. This implies that $H^{r} i_{0}^{!} H^{0} \pi_{+}^{\vee} \mathcal{N}=0$, and $H^{r-1} i_{0}^{!} H^{0} \pi_{+}^{\vee} \mathcal{N}=H^{r} i_{0}^{!} H \leq-1 \pi_{+}^{\vee} \mathcal{N}$. But $H^{\leq-1} \pi_{+}^{\vee} \mathcal{N}$ sits in cohomological degree $\leq-1$ and $i_{0}^{!}$has cohomological amplitude $r, H^{r} i_{0}^{!} H^{\leq-1} \pi_{+}^{\vee} \mathcal{N}=0$.

We can now complete the proof of Theorem 1.9

Proof. Combining (5.3)-(5.5) and Lemma 5.3, we can rewrite (5.1) as

$$
0 \rightarrow \mathcal{O}_{V^{\vee}}^{d} \rightarrow H^{0} \pi_{+}^{\vee} e v^{!}\left(D_{\mathbb{L}^{\vee},-\beta}\right)[1-r] \rightarrow \tau \rightarrow 0 .
$$

Theorem 1.9 follows immediately from Lemma 5.4 and the sequence (5.6).

\section{Rank 1 points of general $G / P$}

In this section, we will describe a rank 1 point for a general homogeneous variety $X$ in the case of $\mathcal{L}=\omega_{X}^{-1}$, using the projected Richardson stratification of $X$ studied in [18, 20, [8] $[13]^{2}$.

We follow the notations in [13]. Let $G$ be a reductive algebraic group over an algebraically closed field $k$ of characteristics zero, $B$ a Borel subgroup and $P \supset B$ a parabolic subgroup

\footnotetext{
${ }^{2}$ We thank T. Lam for pointing out this stratification to us.
} 
An Huang, Bong H. Lian, and Xinwen Zhu

in $G$. Put $B^{+}=B$ and let $B^{-}$be the opposite Borel subgroup. Let $Q\left(W, W_{P}\right)$ be the set of equivalence classes of $P$-Bruhat intervals [13, §2]. Each equivalence class is uniquely specified by a pair $(u, w)$ of elements in the Weyl group. For $(u, w) \in Q\left(W, W_{P}\right)$, put $X_{u}^{w}:=\left(B^{-} u B / B\right) \cap\left(B^{+} w B / B\right)$, an open Richardson variety in $G / B$.

Proposition 6.1. [20, §7][13, §3] There is a stratification of $X=G / P$ of the form $X=$ $\amalg_{(u, w) \in Q\left(W, W_{P}\right)} \stackrel{\circ}{\Pi}_{u}^{w}$, where each stratum $\stackrel{\circ}{\Pi}_{u}^{w}$ is the isomorphic image of $X_{u}^{w}$ under the natural projection $G / B \rightarrow G / P$.

Let $\Pi_{1}, . ., \Pi_{s}$ be the closures of the codimension 1 strata in $X$. By Lemma 5.4 [13], $\cup_{i} \Pi_{i}$ is an anticanonical divisor in $X$. Since $X-\cup_{i} \Pi_{i}$ is the largest stratum, it is isomorphic to an open Richardson variety $X_{u}^{w}$ in $G / B$. It is well-known that (see for example [19])

$$
H_{c}^{N}\left(X_{u}^{w}\right)=\operatorname{Hom}\left(M_{u}, M_{w}\right)
$$

where $N=\operatorname{dim} X_{u}^{w}$ and $M_{w}$ denotes the Verma module of the Lie algebra of $G$ of highest weight $-w(\rho)-\rho$.

By combining Theorems 1-4 [2], or by the Kazhdan-Lusztig conjecture, one has

$$
\operatorname{dim} \operatorname{Hom}\left(M_{u}, M_{w}\right)=1
$$

Therefore, by Theorem 1.4 we have

Corollary 6.2. Let $a \in \Gamma\left(X, \omega_{X}^{-1}\right)$ be the defining section of the anticanonical divisor $\cup_{i} \Pi_{i}$. Then a is a rank 1 point of $X$.

Example 6.3. Consider the Grassmannian $X=G(d, n)$. According to [14, $\cup_{i} \Pi_{i}$ is defined by the section $a=x_{1,2, \ldots, d} x_{2,3, \ldots, d+1} \ldots x_{n, 1, . ., d-1}$, where the $x_{i_{1}, . ., i_{d}}$ are the Plücker coordinates of $X$. This generalizes a construction in [4 for $d=2$.

\section{RANK 1 POINTS OF 1-STEP FLAGS}

Notation. If $m$ is an $p \times q$ matrix, and $J \subset(1,2, . ., p)$ is an ordered index set, then $m_{J}$ denotes the submatrix of $m$ given by the rows labelled by $J$, and we also call $m_{J}$ the $J$-block of $m$. We denote by $x_{J}, J \subset(1,2, . ., n)$, the Plücker coordinates of the $d$-plane Grassmannian $F(d, n)$. Let $M$ be the space of rank $d$ matrices of size $n \times d$. Then $G L_{d}$ acts freely and properly on $M$ by right multiplication and $M / G L_{d} \cong F(d, n)$. Under this identification, we denote the projection map of the Stiefel bundle $M \rightarrow X$ by $m \mapsto[m]:=m \cdot G L_{d}$. Then $x_{J}$ can be viewed as the function $x_{J}: M \rightarrow \mathbb{C}, m \mapsto \operatorname{det}\left(m_{J}\right)$. Given a section $f$ of any line bundle on $X$, we denote by $X(f)$ the complement of $f=0$ in $X$, and by $M(f)$ the preimage of $X(f)$ under $M \rightarrow X$. 
Proposition 7.1. The 1-step flag variety $X=F(d, n)$ admits a rank 1 point $f \in \Gamma\left(X, \omega_{X}^{-1}\right)$ such that $\left(x_{J}\right)^{k} \mid f$ for some $J \subset(1,2, . ., n)$ with $|J|=d$ and $k=\min (d, n-d)$. If $n=2 d$, then $f=\left(x_{1, . ., d}\right)^{d}\left(x_{d+1, . ., n}\right)^{d}$ is a rank 1 point.

Proof. (a) Consider the case $n \geq l+d \geq 2 d$. We have

$$
\begin{aligned}
& X_{1}:=F(d, n-l) \hookrightarrow X, E \mapsto E \oplus 0_{l} \\
& X_{2}:=F(d, l) \hookrightarrow X, E \mapsto 0_{n-l} \oplus E .
\end{aligned}
$$

Here we view $\mathbb{C}^{n}=\mathbb{C}^{n-l} \oplus \mathbb{C}^{l}$. Let $f_{1}$ be a given rank 1 point of $X_{1}$ such that $\left(x_{1, . ., d}\right)^{k_{1}} \mid f_{1}$, $k_{1}=\min (d, n-l-d)$, and $f_{2}$ a rank 1 point of $X_{2}$ such that $\left(x_{n-d+1, . . n}\right)^{k_{2}} \mid f_{2}, k_{2}=$ $\min (d, l-d)$. (In case $l=d, X_{2}=\mathrm{pt}$, we simply take $f_{2}=\left(x_{n-d+1, . ., n}\right)^{d}$; in case $n-l=d$, $X_{1}=$ pt, we take $\left.f_{1}=\left(x_{1, . ., d}\right)^{d}\right)$. We can view $f_{1}, f_{2}$ as sections of $\mathcal{O}_{X}(n-l)$ and $\mathcal{O}_{X}(l)$ respectively on $X=F(d, n)$. Then the restriction of $f_{1}$ to $X_{1}$ under (7.1) becomes a section of $\mathcal{O}_{X_{1}}(n-l)$. Likewise the restriction of $f_{2}$ to $X_{2}$ becomes a section of $\mathcal{O}_{X_{2}}(l)$. We claim that $f=f_{1} f_{2} \in \Gamma\left(X, \omega_{X}^{-1}\right)$ is a rank 1 point of $X$. We will first construct an explicit isomorphism

$$
X_{1}\left(f_{1}\right) \times X_{2}\left(f_{2}\right) \times G L_{d} \rightarrow X(f) .
$$

Let $M_{1}, M_{2}, M$ be the Stiefel bundles over $X_{1}, X_{2}, X$ respectively. Since $x_{J} \mid f_{2}, J=(n-$ $d+1, . . n)$, each $m_{2}^{\prime} \in M_{2}\left(f_{2}\right)$ has a nonsingular $J$-block $D$. Define

$$
M_{1}\left(f_{1}\right) \times M_{2}\left(f_{2}\right) \rightarrow M(f), m_{1}^{\prime}, m_{2}^{\prime} \mapsto m=\left[\begin{array}{c}
m_{1}^{\prime} D \\
m_{2}^{\prime}
\end{array}\right] .
$$

This is well-defined since

$$
f(m)=f_{1}\left(m_{1}^{\prime} D\right) f_{2}\left(m_{2}^{\prime}\right)=(\operatorname{det} D)^{n-l} f_{1}\left(m_{1}^{\prime}\right) f_{2}\left(m_{2}^{\prime}\right) .
$$

The map is a bijection with inverse $m=\left[\begin{array}{l}m_{1} \\ m_{2}\end{array}\right] \mapsto m_{1}\left(m_{2}\right)_{J}^{-1}, m_{2}$. Now let $h \in G L_{d}$ act on $M_{1}\left(f_{1}\right) \times M_{2}\left(f_{2}\right)$ by the formula $\left(m_{1}^{\prime}, m_{2}^{\prime} h^{-1}\right)$. Then the map is equivariant. It follows that we have an isomorphism

$$
M_{1}\left(f_{1}\right) \times X_{2}\left(f_{2}\right) \rightarrow X(f) .
$$

Finally, since $x_{1, . ., d} \mid f_{1}$ each $m_{1}^{\prime} \in M_{1}\left(f_{1}\right)$ has a nonsingular top $d \times d$ block. It follows that the principal bundle $G L_{d}-M_{1}\left(f_{1}\right) \rightarrow X_{1}\left(f_{1}\right)$ is trivial. In fact, it has a (unique) section of the form $\left[m_{1}\right] \mapsto m_{1}^{\prime}$ where $m_{1}^{\prime}$ is the unique representative in $\left[m_{1}\right]$ whose top $d \times d$ block is the identity matrix $I_{d}$. This proves that

$$
X(f) \cong X_{1}\left(f_{1}\right) \times X_{2}\left(f_{2}\right) \times G L_{d} .
$$

Since the $X_{i}\left(f_{i}\right)$ are affine varieties, all de Rham cohomology of degree $>\operatorname{dim} X_{i}$, vanishes. Since $f_{1}, f_{2}$ are rank 1 points of $X_{1}, X_{2}$ respectively, we have $H^{\operatorname{dim} X_{i}}\left(X_{i}\left(f_{i}\right)\right)=\mathbb{C}$ by Theorem 1.4. It follows that

$$
H^{\operatorname{dim} X}(X(f)) \cong H^{\operatorname{dim} X_{1}}\left(X_{1}\left(f_{1}\right)\right) \otimes H^{\operatorname{dim} X_{2}}\left(X_{2}\left(f_{2}\right)\right) \otimes H^{d^{2}}\left(G L_{d}\right) \cong \mathbb{C} .
$$

So $f$ is a rank 1 point of $X$ such that $\left(x_{1, . ., d}\right)^{k_{1}}\left(x_{n-d+1, . . n}\right)^{k_{2}} \mid f$. 
(b) To complete the proof of the proposition, we proceed by induction. For $X=F(1,2)=$ $\mathbb{P}^{1}$, paragraph (a) with $n=2$ and $l=d=1$ shows that $x_{1} x_{2}$ is a rank 1 point of $X$, and the proposition holds. Assume that it holds for up to $F(d, n-1)$, and consider the case $X=F(d, n)$. For $n<2 d$ we have $F(d, n) \cong F(n-d, n)$, in which case paragraph (a) with $l, d$ playing the role of $d, n-d$, yields a rank 1 point $f$ of $F(n-d, n)$ with $\left(x_{J}\right)^{n-d} \mid f$ and $|J|=n-d$. This in turn yields a rank 1 point of $F(d, n)$ divisible by $\left(x_{J^{c}}\right)^{n-d}$ where $J^{c}=(1, . ., n)-J$. For $n=2 d$, paragraph (a) with $n=l+d=2 d$ shows that $\left(x_{1, \ldots, d}\right)^{d}\left(x_{d+1, \ldots, n}\right)^{d}$ is a rank 1 point of $X$. For $n>2 d$, paragraph (a) with $l=d$ and our inductive hypothesis shows that $X$ has a rank 1 point $f=f_{1} \cdot\left(x_{n-d+1, \ldots, n}\right)^{d}$, where $f_{1}$ is a rank 1 point of $F(d, n-d)$. This completes the proof.

Corollary 7.2. Let $n=l_{1}+\cdots+l_{s}$ be a partition of $n$ with $l_{p} \geq d$. Let $f_{p}$ be a rank 1 point of $F\left(d, l_{p}\right) \hookrightarrow F(d, n)$, viewed as a degree $l_{p}$ polynomial in the Plücker coordinates $x_{J}$ of $X=F(d, n)$ with $J \subset\left(l_{1}+\cdots+l_{p-1}+1, . ., l_{1}+\cdots+l_{p}\right)$ and $|J|=d$, such that $\left(x_{l_{1}+\cdots+l_{p-1}+1, . ., l_{1}+\cdots+l_{p-1}+d}\right) \mid f_{p}$. Then $f=f_{1} \cdots f_{s}$ is a rank 1 point of $X$. In fact, we have an isomorphism

$$
X(f) \cong X_{1}\left(f_{1}\right) \times \cdots \times X_{s}\left(f_{s}\right) \times\left(G L_{d}\right)^{s-1}
$$

where $X_{p}:=F\left(d, l_{p}\right)$.

Proof. Start with $l=l_{2}+\cdots+l_{s}$. Then paragraph (a) in the preceding proof gives

$$
X(f) \cong X_{1}\left(f_{1}\right) \times X_{2}^{\prime}\left(f_{2} \cdots f_{s}\right) \times G L_{d}
$$

where $X_{2}^{\prime}:=F\left(d, n-l_{1}\right)$. Now the result follows by induction on $s$.

\section{RANK 1 POINTS OF $r$-STEP FLAGS}

Throughout this section, let $X=F\left(d_{1}, . ., d_{r}, n\right)$ be the $r$-step flag variety with $r \geq 2$. We will give a recursive procedure that produces a rank 1 point of $X$, by assembling rank 1 points of lower step flag varieties. We begin with some notations and terminology.

Let $\mathcal{O}_{i}(1)$ be the standard hyperplane bundle on $F\left(d_{i}, n\right)$. The space of its sections is an irreducible $G=S L_{n}$ module of highest weight $\lambda_{d_{i}}$, the $d_{i}$ th fundamental weight of $G$. We shall denote by $\lambda_{d_{i}}$ the pullback of $\mathcal{O}_{i}(1)$ via the composition map

$$
X \hookrightarrow F\left(d_{1}, n\right) \times \cdots \times F\left(d_{r}, n\right) \rightarrow F\left(d_{i}, n\right)
$$

where the first is the incidence embedding and the second is the $i$ th projection. Then Pic $(X)$ is the free abelian group generated by $\lambda_{d_{1}}, \ldots, \lambda_{d_{r}}$. We also have (see [16])

$$
-K_{X}=\omega_{X}^{-1}=\left(n-d_{r-1}\right) \lambda_{d_{r}}+\left(d_{r}-d_{r-2}\right) \lambda_{d_{r-1}}+\cdots+\left(d_{3}-d_{1}\right) \lambda_{d_{2}}+d_{2} \lambda_{d_{1}}
$$


By the Borel-Weil theorem, the restriction map

$$
\Gamma\left(F\left(d_{1}, n\right), \mathcal{O}_{1}\left(k_{1}\right)\right) \otimes \cdots \otimes \Gamma\left(F\left(d_{r}, n\right), \mathcal{O}_{r}\left(k_{r}\right)\right) \rightarrow \Gamma\left(X, \sum_{i} k_{i} \lambda_{d_{i}}\right)
$$

is a $G$-equivariant surjective map for any $k_{1}, . ., k_{r} \in \mathbb{Z}$ (and both spaces are zero unless $k_{i} \geq 0$ for all $i$ ). Thus any homogeneous polynomial in the Plücker coordinates $x_{J_{i}}$ with $\left|J_{i}\right|=d_{i}$, of multi-degree $\left(k_{1}, . ., k_{r}\right) \in \mathbb{Z}_{\geq 0}^{r}$, can be viewed as a section of the line bundle $\sum_{i} k_{i} \lambda_{d_{i}}$ on $X$. Conversely, any section of this line bundle on $X$ can be expressed as such a polynomial (not necessarily unique).

Let $k<n-d_{r}$ and consider the embeddings

$$
\begin{aligned}
& X_{1}:=F\left(d_{1}, . ., d_{r}, n-k\right) \hookrightarrow X, E^{\bullet} \mapsto E^{\bullet} \oplus 0_{k} \\
& X_{2}:=F\left(d_{1}-k, . ., d_{r}-k, n-k\right) \hookrightarrow X, E^{\bullet} \mapsto E^{\bullet} \oplus \mathbb{C}^{k} .
\end{aligned}
$$

Here we view $\mathbb{C}^{n}=\mathbb{C}^{n-k} \oplus \mathbb{C}^{k}$, and $X_{1}, X_{2}$ are viewed as spaces consisting of $r$-step flags in the factor $\mathbb{C}^{n-k}$. For each Plücker coordinate $x_{J^{\prime}}$ on $X_{1}$ with $J^{\prime} \subset(1,2, \ldots, n-k)$, is the restriction of $x_{J^{\prime}}$, regarded as a Plücker coordinate on $X$. Likewise, any homogeneous polynomial $f_{1}$ in the $x_{J^{\prime}}$, can be viewed as the restriction of a section $\bar{f}_{1}$ on $X$ involving only the same Plücker coordinates. We shall often impose certain divisibility conditions (called the hyperplane property - see below) on $\bar{f}_{1}$, but will state them in terms of $f_{1}$. Similarly each Plücker coordinate $x_{J^{\prime}}$ on $X_{2}$ is the restriction of $x_{J^{\prime} \cup(n-k+1, . ., n)}$ on $X$; any given homogeneous polynomial $f_{2}$ in the $x_{J^{\prime}}$, is the restriction of a section $\tilde{f}_{2}$ on $X$ involving only the $x_{J^{\prime} \cup(n-k+1, . ., n)}$. Again, divisibility conditions imposed on $\tilde{f}_{2}$ will be stated in terms of $f_{2}$.

As in the case of 1-step flags, we can view $X=M / H$, where

$$
H:=G L_{d_{r}} \times \cdots \times G L_{d_{1}}
$$

and $M$ is the space of $r$-tuple of matrices $m=\left(m_{r}, . ., m_{1}\right), m_{i}$ a $d_{i+1} \times d_{i}$ matrix of rank $d_{i}$ $\left(d_{r+1} \equiv n\right)$, where $h=\left(h_{r}, . ., h_{1}\right) \in H$ acts on $M$ by the formula

$$
m \cdot h^{-1}:=\left(m_{r} h_{r}^{-1}, h_{r} m_{r-1} h_{r-1}^{-1}, . ., h_{2} m_{1} h_{1}^{-1}\right) .
$$

Under the identification $X=M / H$, we denote the projection map $M \rightarrow X$ by $m \mapsto[m]:=$ $m \cdot H$, and call $M$ the Stiefel bundle over $X$. We can view a Plücker coordinate $x_{J},|J|=d_{i}$, on $X$ as the function $x_{J}: M \rightarrow \mathbb{C}, x_{J}(m)=\operatorname{det}\left(m_{r} \cdots m_{i}\right)_{J}$. In particular, $f_{1}$ is a section on $X_{1}$ and $\bar{f}_{1}$ a section on $X$ restricting to it as described above, then for $J=(1, . ., n-k)$ we have

$$
\bar{f}_{1}\left(m_{r}, . ., m_{1}\right)=f_{1}\left(\left(m_{r}\right)_{J}, m_{r-1}, . ., m_{1}\right)
$$

whenever $\operatorname{rk}\left(m_{r}\right)_{J}=d_{r}$. Let $m=\left(m_{r}, . ., m_{1}\right) \in M$ where the $m_{i}$ have the form

$$
m_{i}=\left[\begin{array}{cc}
m_{i}^{\prime} & * \\
O & I_{k}
\end{array}\right]
$$


where $I_{k}$ is the $k \times k$ identity matrix and $O$ a zero block. Then $x_{J^{\prime} \cup(n-k+1, . ., n)}(m)=$ $\operatorname{det}\left(m_{r}^{\prime} \cdots m_{i}^{\prime}\right)_{J^{\prime}}$ for any $J^{\prime} \subset(1, . ., n-k)$ with $\left|J^{\prime}\right|=d_{i}-k$. So, if $f_{2}$ is a section on $X_{2}$ and $\tilde{f}_{2}$ a section on $X$ restricting to it as described above, then

$$
\tilde{f}_{2}\left(m_{r}, . ., m_{1}\right)=f_{2}\left(m_{r}^{\prime}, \ldots, m_{1}^{\prime}\right) .
$$

Let $f$ be a nonzero section of a line bundle on $X$, and let $X(f)$ be the complement of $f=0$, and $M(f)$ the preimage of $X(f)$ under $M \rightarrow X$.

Definition 8.1. (Hyperplane property) We say that $f$ has the hyperplane property if for some $J_{i} \subset(1,2, . ., n)$ with $\left|J_{i}\right|=d_{i}, i=1, . ., r$, we have $\left(x_{J_{1}} \cdots x_{J_{r}}\right) \mid f$. In other words, the hypersurface $f=0$ contains the union of hyperplanes $x_{J_{i}}=0$.

Note that if $f$ has the hyperplane property, we can always find a suitable permutation matrix $g \in G L_{n}$ such that the $g$-translate of $f$ has the hyperplane property where $J_{r}=$ $\left(n-d_{r}+1, . ., n\right)$. In the construction that follows, we will often arrange our section $f$ so that this occurs. Next, we have the following elementary lemma.

Lemma 8.1. Assume $f$ has the hyperplane property $\left(x_{J_{1}} \cdots x_{J_{r}}\right) \mid f$. Then the principal $H$-bundle $M(f) \rightarrow X(f)$, has a unique section $m=\left(m_{r}, . ., m_{1}\right)$, where the $m_{r}, . ., m_{1}$ are matrix valued functions on $X(f)$ such that

$$
\left(m_{r} \cdots m_{i}\right)_{J_{i}}=I_{d_{i}} .
$$

Definition 8.2. (Special section) We call the section given in Lemma 8.1 the special section of $M(f)$ (which depends on the index sets $J_{1}, . ., J_{r}$ ).

We now describe our recursive procedure that produces a rank 1 point of $X$ with the hyperplane property.

Case 1. Assume $d_{r-1}+d_{r}<n$. Consider (cf. [8.2)

$$
\begin{aligned}
& X_{1}:=F\left(d_{1}, . ., d_{r-1}, d_{r}\right) \hookrightarrow F\left(d_{1}, . ., d_{r-1}, n\right), E^{\bullet} \mapsto 0_{n-d_{r}} \oplus E^{\bullet} \\
& X_{2}:=F\left(d_{r}, n\right) .
\end{aligned}
$$

Let $M_{1}, M_{2}, M$ be the Stiefel bundles over $X_{1}, X_{2}, X$ respectively. Let $f_{1}, f_{2}$ be rank 1 points of $X_{1}, X_{2}$ respectively with the hyperplane properties

$$
\left(x_{J_{1}} \cdots x_{J_{r-1}}\right)\left|f_{1},\left(x_{J_{r}}\right)^{k}\right| f_{2}
$$

for some $J_{i}$ with $\left|J_{i}\right|=d_{i}, i=1, \ldots, r$, and $J_{1}=\left(1, . ., d_{1}\right), J_{r}=\left(n-d_{r}+1, . ., n\right), k=$ $\min \left(d_{r}, n-d_{r}\right)>d_{r-1}$. Such an $f_{2}$ exists by Proposition 7.1 Put

$$
f=\bar{f}_{1} \cdot \bar{f}_{2} \cdot\left(x_{J_{r}}\right)^{-d_{r-1}} .
$$


Then we have

$$
\left(x_{J_{1}} \cdots x_{J_{r}}\right) \mid f
$$

It follows easily from (8.1) that $f$ is a section of $\omega_{X}^{-1}$.

Lemma 8.2. We have an $H=G L_{d_{r}} \times \cdots \times G L_{d_{1}}$ equivariant isomorphism

$$
\begin{aligned}
& M_{1}\left(f_{1}\right) \times M_{2}\left(f_{2}\right) \rightarrow M(f) \\
& \left(m_{r-1}^{\prime}, . ., m_{1}^{\prime}\right), m_{r}^{\prime} \mapsto m=\left(m_{r}^{\prime}, D^{-1} m_{r-1}^{\prime}, m_{r-2}^{\prime}, . ., m_{1}^{\prime}\right)
\end{aligned}
$$

where $D$ is the $J_{r}$-block of $m_{r}^{\prime}$. Therefore the map descends to an isomorphism $X_{1}\left(f_{1}\right) \times$ $X_{2}\left(f_{2}\right) \rightarrow X(f)$.

Proof. For $m_{r}^{\prime} \in M_{2}\left(f_{2}\right)$, its $J_{r}$-block $D$ is a nonsingular matrix in $G L_{d_{2}}$ since $\left(x_{J_{r}}\right)^{k} \mid f_{2}$. Suppose $f_{1}\left(m_{r-1}^{\prime}, . ., m_{1}^{\prime}\right) f_{2}\left(m_{r}^{\prime}\right) \neq 0$. Then

$$
f(m)=f_{1}\left(\left(m_{r}^{\prime} D^{-1} m_{r-1}^{\prime}\right)_{J_{r}}, m_{r-2}^{\prime}, . ., m_{1}^{\prime}\right) f_{2}\left(m_{r}^{\prime}\right)\left(\operatorname{det}\left(m_{r}^{\prime}\right)_{J_{r}}\right)^{-d_{r-1}} .
$$

Since $\left(m_{r}^{\prime}\right)_{J_{r}}=D$, it follows that $\left(m_{r}^{\prime} D^{-1} m_{r-1}^{\prime}\right)_{J_{r}}=m_{r-1}^{\prime}$ and we have

$$
f(m)=f_{1}\left(m_{r-1}^{\prime}, . ., m_{1}^{\prime}\right) f_{2}\left(m_{r}^{\prime}\right)(\operatorname{det} D)^{-d_{r-1}} \neq 0 .
$$

So, the map is well-defined. Now, $h=\left(h_{r}, . ., h_{1}\right) \in H$ acts on $M(f)$ by (8.3), and on $M_{1}\left(f_{1}\right) \times M_{2}\left(f_{2}\right)$ by the formula

$$
m_{r} h_{r}^{-1},\left(m_{r-1} h_{r-1}^{-1}, h_{r-1} m_{r-2} h_{r-2}^{-1}, . ., h_{2} m_{1} h_{1}^{-1}\right) .
$$

Therefore our map is $H$-equivariant. Moreover, the map

$$
M(f) \rightarrow M_{1}\left(f_{1}\right) \times M_{2}\left(f_{2}\right),\left(m_{r}, . ., m_{1}\right) \mapsto\left(\left(m_{r}\right)_{J_{r}} m_{r-1}, m_{r-2}, . ., m_{1}\right), m_{r}
$$

is well-defined and is the inverse of the map above.

The lemma and Theorem 1.4 imply

Proposition 8.3. For $d_{r-1}+d_{r}<n$, if any $s$-step flag variety for $s<r$ admits a rank 1 point with the hyperplane property, then $X=F\left(d_{1}, . ., d_{r}, n\right)$ admits one as well.

By Proposition [7.1] for $d_{1}+d_{2}<n$ it follows that $F\left(d_{1}, d_{2}, n\right)$ admits a rank 1 point with the hyperplane property. This also implies that for $d_{1}+d_{2}>n$, then $F\left(d_{1}, d_{2}, n\right) \cong$ $F\left(n-d_{2}, n-d_{1}, n\right)$ admits one as well.

Case 2. Assume $d_{r-1}+d_{r}=n$ and $r=2$. Consider the following section of $\omega_{X}^{-1}$ :

$$
f=\left(x_{1, . ., d_{1}}\right)^{d_{2}}\left(x_{d_{1}+1, \ldots, n}\right)^{d_{2}} .
$$

Then by Lemma 8.1, the special section of $M(f) \rightarrow X(f)$ has the form

$$
m=\left(m_{2}, m_{1}\right)=\left(\left[\begin{array}{c}
A_{2} \\
I_{d_{2}}
\end{array}\right], m_{1}\right) \text { such that } A_{2} m_{1}=I_{d_{1}} .
$$


Since $m_{1}(o)$ has rank $d_{1}$ at each point $o \in X(f)$, the second equation shows that the function

$$
m_{1}: X(f) \rightarrow M_{1}, o \mapsto m_{1}(o)
$$

is onto. Here $M_{1}$ be the Stiefel bundle over $F\left(d_{1}, d_{2}\right)$. Moreover, the level set of this function at each point is an affine space of dimension $d_{1} d_{2}-d_{1}^{2}$. It follows that $X(f)$ is homotopy equivalent to $M_{1}$. Finally, the principal $G L_{d_{1}}$-bundle $M_{1} \rightarrow F\left(d_{1}, d_{2}\right)$ is over a simply connected base. Thus by the Serre spectral sequence, the highest degree nonzero cohomology group of $M_{1}$ is one dimensional at degree $2 d_{1} d_{2}-d_{1}^{2}=\operatorname{dim} X$. By Theorem 1.4, we have

Proposition 8.4. For $d_{1}+d_{2}=n, X=F\left(d_{1}, d_{2}, n\right)$ admits the rank 1 point $f=$ $\left(x_{1, . ., d_{1}}\right)^{d_{2}}\left(x_{d_{1}+1, \ldots, n}\right)^{d_{2}}$.

Remark 8.1. The propositions in Cases 1-2 $(r=2)$ now imply that any 2 -step flag variety $F\left(d_{1}, d_{2}, n\right)$ admits a rank 1 point with the hyperplane property.

Case 3. Assume $d_{r-1}+d_{r}=n$ and $r \geq 3$. Consider (cf. (8.2) )

$$
\begin{aligned}
& X_{1}:=F\left(d_{1}, . ., d_{r-2}, d_{r-1}\right) \hookrightarrow F\left(d_{1}, . ., d_{r-2}, n\right), E^{\bullet} \mapsto E^{\bullet} \oplus 0_{n-d_{r-1}} \\
& X_{2}:=F\left(d_{r-1}, d_{r}, n\right) .
\end{aligned}
$$

Let $M_{1}, M_{2}, M$ be the Stiefel bundles over $X_{1}, X_{2}, X$ respectively. Let $f_{1}, f_{2}$ be rank 1 points of $X_{1}, X_{2}$ respectively with the hyperplane properties

$$
\left(x_{J_{1}} \cdots x_{J_{r-2}}\right) \mid f_{1}, f_{2}=\left(x_{J_{r-1}}\right)^{d_{r}}\left(x_{J_{r}}\right)^{d_{r}}
$$

for some $J_{i}$ with $\left|J_{i}\right|=d_{i}, i=1, \ldots, r$, and $J_{1}=\left(1, . ., d_{r-1}\right), J_{r}=\left(n-d_{r}+1, . ., n\right)$. Note that $f_{2}$ is given by Proposition 8.4 Put

$$
f=\bar{f}_{1} \cdot \bar{f}_{2} \cdot\left(x_{J_{r-1}}\right)^{-d_{r-2}} \in \Gamma\left(X, \omega^{-1}\right) .
$$

Then we have

$$
\left(x_{J_{1}} \cdots x_{J_{r-1}}\left(x_{J_{r}}\right)^{d_{r}}\right) \mid f
$$

Since $x_{J_{r-1}} \mid f_{2}$, the $J_{r-1}=\left(1, . ., d_{r-1}\right)$-block $D$ of $m_{r}^{\prime} m_{r-1}^{\prime}$ for $\left(m_{r}^{\prime}, m_{r-1}^{\prime}\right) \in M_{2}\left(f_{2}\right)$ is nonsingular.

Lemma 8.5. We have an $H=G L_{d_{r}} \times \cdots \times G L_{d_{1}}$ equivariant isomorphism

$$
\begin{aligned}
& M_{1}\left(f_{1}\right) \times M_{2}\left(f_{2}\right) \rightarrow M(f) \\
& \left(m_{r-2}^{\prime}, . ., m_{1}^{\prime}\right),\left(m_{r}^{\prime}, m_{r-1}^{\prime}\right) \mapsto m=\left(m_{r}^{\prime}, m_{r-1}^{\prime}, D^{-1} m_{r-2}^{\prime}, m_{r-3}^{\prime}, . ., m_{1}^{\prime}\right)
\end{aligned}
$$

where $D$ is the $J_{r-1}=\left(1, . ., d_{r-1}\right)$-block of $m_{r}^{\prime} m_{r-1}^{\prime}$. Therefore the map descends to an isomorphism $X_{1}\left(f_{1}\right) \times X_{2}\left(f_{2}\right) \rightarrow X(f)$. 
The proof is closely analogous to the lemma in Case 1, and will be omitted. The lemma and Theorem 1.4 imply

Proposition 8.6. For $d_{r-1}+d_{r}=n$, if any s-step flag variety for $s<r$ admits a rank 1 point with the hyperplane property, then $X=F\left(d_{1}, . ., d_{r}, n\right)$ admits one such $f$ that satisfies $\left(x_{J_{r}}\right)^{d_{r}} \mid f$ where $J_{r}=\left(n-d_{r}+1, . ., n\right)$.

Case 4. Assume $d_{1}+d_{2}=n$. Then $X \cong F\left(n-d_{r}, . ., n-d_{2}, n-d_{1}, n\right)$, which belongs in Case 3, and the analogue of Proposition 8.6 is

Proposition 8.7. For $d_{1}+d_{2}=n$, if any $s$-step flag variety for $s<r$ admits a rank 1 point with the hyperplane property, then $X=F\left(d_{1}, . ., d_{r}, n\right)$ admits one such $f$ that satisfies $\left(x_{J_{1}}\right)^{d_{1}} \mid f$ where $J_{1}=\left(1, . ., d_{1}\right)$.

Case 5. Assume $d_{r-1}+d_{r}>n$. There exists a unique $a$ with $r>a>1$ such that $d_{a}+d_{a+1}>n \geq d_{a-1}+d_{a}$. Assume $n>2 d_{a}$ first. We will consider $n=2 d_{a}$ and $n=d_{a-1}+d_{a}$ in Cases 6-7 below separately. Consider

$$
\begin{aligned}
& X_{1}:=F\left(d_{1}, . ., d_{a}, n-d_{a}\right) \hookrightarrow F\left(d_{1}, . ., d_{a}, n\right),\left(E_{1}^{i}\right) \mapsto\left(E_{1}^{i} \oplus 0_{d_{a}}\right) \\
& X_{2}:=F\left(d_{a+1}-d_{a}, . ., d_{r}-d_{a}, n-d_{a}\right) \hookrightarrow F\left(d_{a+1}, . ., d_{r}, n\right),\left(E_{2}^{j}\right) \mapsto\left(E_{2}^{j} \oplus \mathbb{C}^{d_{a}}\right) .
\end{aligned}
$$

Here we view $\mathbb{C}^{n}=\mathbb{C}^{n-d_{a}} \oplus \mathbb{C}^{d_{a}}$. Let $M_{1}, M_{2}, M$ be the Stiefel bundles over $X_{1}, X_{2}, X$ respectively. Let $f_{1}, f_{2}$ be rank 1 points of $X_{1}, X_{2}$ respectively with the hyperplane properties

$$
\left(x_{J_{1}} \cdots x_{J_{a}}\right)\left|f_{1},\left(x_{J_{a+1}^{\prime}} \cdots x_{J_{r}^{\prime}}\right)\right| f_{2}
$$

for some $J_{i} \subset\left(1,2, . ., n-d_{a}\right)$ with $\left|J_{i}\right|=d_{i}(i=1, \ldots, a)$ and $J_{a}=\left(n-2 d_{a}+1, . ., n-d_{a}\right)$, and for some $J_{i}^{\prime} \subset\left(1,2, . ., n-d_{a}\right)$ with $\left|J_{i}^{\prime}\right|=d_{i}-d_{a}(i=a+1, . ., r)$ and $J_{r}^{\prime}=\left(n-d_{r}+1, . ., n-d_{a}\right)$. Put $J:=\left(n-d_{a}+1, . ., n\right), J_{i}:=J_{i}^{\prime} \cup J, i=a+1, . ., n$, and

$$
f:=\bar{f}_{1} \cdot \tilde{f}_{2} \cdot\left(x_{J}\right)^{d_{a+1}+d_{a}-n} .
$$

Then $f$ has the hyperplane property

$$
\left(x_{J_{1}} \cdots \widehat{x_{J_{a}}} \cdots x_{J_{r}} x_{J}\right) \mid f .
$$

Lemma 8.8. The special section $m=\left(m_{r}, . ., m_{1}\right)$ (cf. Lemma 8.1) of $M(f) \rightarrow X(f)$ has the following form:

$$
\begin{aligned}
m_{i} & =\left[\begin{array}{cc}
m_{i}^{\prime} & A_{i} \\
O & I_{d_{a}}
\end{array}\right], i=a+1, . ., r \\
m_{a} & =\left[\begin{array}{c}
A_{a} \\
I_{d_{a}}
\end{array}\right] \\
m_{r} \cdots m_{a} & =\left[\begin{array}{c}
m_{a}^{\prime} D \\
I_{d_{a}}
\end{array}\right] \\
m_{a-1} & =D^{-1} m_{a-1}^{\prime} \\
m_{i} & =m_{i}^{\prime}, \quad i=1, . ., a-2
\end{aligned}
$$


where $D$ is a $G L_{d_{a}}$-valued function, $A_{a}, . ., A_{r}$ are matrix valued functions, and $\left(m_{a}^{\prime}, . ., m_{1}^{\prime}\right)$, $\left(m_{r}^{\prime}, . ., m_{a+1}^{\prime}\right)$ are matrix valued functions taking values in the special sections of the $M_{1}\left(f_{1}\right) \rightarrow$ $X_{1}\left(f_{1}\right), M_{2}\left(f_{2}\right) \rightarrow X_{2}\left(f_{2}\right)$ respectively.

Proof. For $o \in X(f)$, we will write $m_{i} \equiv m_{i}(o), m_{i}^{\prime} \equiv m_{i}^{\prime}(o), D \equiv D(o)$, etc. Then $m=m(o) \in M(f)$ means that

$$
0 \neq f(m)=\bar{f}_{1}\left(m_{r} \cdots m_{a}, m_{a-1}, . ., m_{1}\right) \tilde{f}_{2}\left(m_{r}, . ., m_{a+1}\right) \operatorname{det}\left(m_{r}\right)_{J_{r}} .
$$

(a) Since $x_{J_{r}^{\prime}} \mid f_{2}$, we have $x_{J_{r}} \mid \tilde{f}_{2}$, and so our $m_{r}$ has the correct form, i.e. $\left(m_{r}\right)_{J_{r}}=I_{d_{r}}$ (hence $\operatorname{det}\left(m_{r}\right)_{J_{r}}=1$ ), and $\left(m_{r}^{\prime}\right)_{J_{r}^{\prime}}=I_{d_{r}-d_{a}}$. Since $\left(x_{J_{a+1}^{\prime}} \cdots x_{J_{r}^{\prime}}\right) \mid f_{2}$, we have $\left(x_{J_{a+1}} \cdots x_{J_{r}}\right) \mid \tilde{f}_{2}$, hence $\left(m_{r} \cdots m_{i}\right)_{J_{i}}=I_{d_{i}}$. By induction on $i$, it is easy to see that our $m_{r}, . ., m_{i}$ above have the correct form, so that

$$
m_{r} \cdots m_{i}=\left[\begin{array}{cc}
m_{r}^{\prime} \cdots m_{i}^{\prime} & * \\
O & I_{d_{a}}
\end{array}\right]
$$

and that $\left(m_{r}^{\prime} \cdots m_{i}^{\prime}\right)_{J_{i}^{\prime}}=I_{d_{i}-d_{a}}$ for $i=a+1 . ., r$. This shows that $\left(m_{r}^{\prime}, . ., m_{a+1}^{\prime}\right)$ actually lies in the special section of $M_{2}\left(f_{2}\right) \rightarrow X_{2}\left(f_{2}\right)$, as asserted.

(b) Since $x_{J} \mid f$, we have $\left(m_{r} \cdots m_{a}\right)_{J}=I_{d_{a}}$. From (8.14), it follows that $\left(m_{a}\right)_{J}=$ $I_{d_{a}}$. Since $x_{J_{a}} \mid f_{1}$, hence $x_{J_{a}} \mid f$, it follows that $\left(m_{r} \cdots m_{a}\right)_{J_{a}}$ is a nonsingular matrix $D \in$ $G L_{d_{a}}$. Thus $m_{a}$ has the correct form as asserted, and $\left(m_{a}^{\prime}\right)_{J_{a}}=I_{d_{a}}$. This also shows that $\left(m_{r} \cdots m_{a}\right)_{1,2, \ldots, n-d_{a}}=m_{a}^{\prime} D$ has rank $d_{a}$, hence

$$
0 \neq \bar{f}_{1}\left(m_{r} \cdots m_{a}, m_{a-1}, . ., m_{1}\right)=f_{1}\left(m_{a}^{\prime} D, m_{a-1}, . ., m_{1}\right) .
$$

Since $f_{1}$ is $G L_{d_{a}}$-equivariant, this is equivalent to

$$
0 \neq f_{1}\left(m_{a}^{\prime}, D^{-1} m_{a-1}, m_{a-2}, . ., m_{1}\right) .
$$

This implies that

$$
\left(m_{a}^{\prime}, m_{a-1}^{\prime}, . ., m_{1}^{\prime}\right)=\left(m_{a}^{\prime}, D^{-1} m_{a-1}, m_{a-2}, . ., m_{1}\right)
$$

lies in the special section of $M_{1}\left(f_{1}\right) \rightarrow X_{1}\left(f_{1}\right)$, as asserted.

This completes the proof.

We now use the special section $m: X(f) \rightarrow M(f)$ described in the preceding lemma to define a map

$$
\begin{aligned}
& X(f) \rightarrow X_{1}\left(f_{1}\right) \times X_{2}\left(f_{2}\right) \times G L_{d_{a}} \\
& o \mapsto\left[m_{a}^{\prime}(o), . ., m_{1}^{\prime}(o)\right],\left[m_{r}^{\prime}(o), . ., m_{a+1}^{\prime}(o)\right], D(o) .
\end{aligned}
$$

We will prove that this is an isomorphism. We will need the following elementary lemma. 
Lemma 8.9. Let $m_{2}^{\prime}$ be a $\left(n-d_{1}\right) \times\left(d-d_{1}\right)$ matrix, and $A_{1}, A_{2}$ be $\left(d-d_{1}\right) \times d_{1}$ and $\left(n-d_{1}\right) \times d_{1}$ matrices. Put

$$
m_{2}=\left[\begin{array}{cc}
m_{2}^{\prime} & A_{2} \\
O & I_{d_{1}}
\end{array}\right], m_{1}=\left[\begin{array}{c}
A_{1} \\
I_{d_{1}}
\end{array}\right]
$$

and assume that $J^{\prime} \subset\left(1, . ., n-d_{1}\right),\left|J^{\prime}\right|=d_{2}-a_{1}$, and that the $J=J^{\prime} \cup\left(n-d_{1}+1, . ., n\right)$-block of $m_{2}$ is $I_{d}$ (which is equivalent to that $\left(A_{2}\right)_{J^{\prime}}=O$ and $\left(m_{2}^{\prime}\right)_{J^{\prime}}=I_{d-d_{1}}$ ). Then $A_{1}, A_{2}$ can be uniquely expressed as polynomial functions in terms of $m_{2}^{\prime}$ and $m_{2} m_{1}$.

Lemma 8.10. The map 8.15 is an isomorphism.

Proof. We will explicitly construct the inverse of [8.15). It is enough to show that given a point $m^{\prime}:=\left(\left(m_{a}^{\prime}, . ., m_{1}^{\prime}\right),\left(m_{r}^{\prime}, . ., m_{a+1}^{\prime}\right), D\right)$ in the special section of the bundle $M_{1}\left(f_{1}\right) \times$ $M_{2}\left(f_{2}\right) \times G L_{d_{a}} \rightarrow X_{1}\left(f_{1}\right) \times X_{2}\left(f_{2}\right) \times G L_{d_{a}}$, the relations (8.13) uniquely determine a point $m=\left(m_{r}, . ., m_{1}\right) \in M$, expressible polynomially in terms of $m^{\prime}$. In fact, it is enough to show that the $A_{a}, . ., A_{r}$ can be so-expressed. Note that the relations (8.13) ensures that $m$ lies in the special section of the bundle $M(f) \rightarrow X(f)$.

By (8.13), we have for $i=1, . ., a+1$,

$$
m_{r} \cdots m_{i}=\left[\begin{array}{cc}
m_{r}^{\prime} \cdots m_{i}^{\prime} & m_{r}^{\prime} \cdots m_{i+1}^{\prime} A_{i}+\cdots+m_{r}^{\prime} A_{r-1}+A_{r} \\
O & I_{d_{a}}
\end{array}\right] .
$$

Since $m_{a}=\left[\begin{array}{c}A_{a} \\ I_{d_{a}}\end{array}\right]$, Lemma 8.9 implies that $A_{a}$ and $m_{r}^{\prime} \cdots m_{a+2}^{\prime} A_{a+1}+\cdots+m_{r}^{\prime} A_{r-1}+A_{r}$ can be uniquely expressed polynomially in terms of $m^{\prime}$. It follows that the right hand block of $m_{r} \cdots m_{a+1}$ :

$$
\begin{aligned}
& \left(m_{r} \cdots m_{a+1}\right)_{R}=\left[\begin{array}{c}
m_{r}^{\prime} \cdots m_{a+2}^{\prime} A_{a+1}+\cdots+m_{r}^{\prime} A_{r-1}+A_{r} \\
I_{d_{a}}
\end{array}\right] \\
& =\left[\begin{array}{cc}
m_{r}^{\prime} \cdots m_{a+2}^{\prime} & m_{r}^{\prime} \cdots m_{a+1}^{\prime} A_{a+2}+\cdots+m_{r}^{\prime} A_{r-1}+A_{r} \\
O & I_{d_{a}}
\end{array}\right]\left[\begin{array}{c}
A_{a+1} \\
I_{d_{a}}
\end{array}\right] \\
& =m_{r} \cdots m_{a+2}\left[\begin{array}{c}
A_{a+1} \\
I_{d_{a}}
\end{array}\right]
\end{aligned}
$$

can be so-expressed. By Lemma 8.9 again, the right hand block of $m_{r} \cdots m_{a+2}$ and $A_{a+1}$ can also be so-expressed. Continuing this way, we see that $A_{a}, . ., A_{r}$ all can be so-expressed. This completes the proof.

The lemma and Theorem 1.4 imply

Proposition 8.11. For $d_{a}+d_{a+1}>n>2 d_{a}$ with $r>a>1$, if any s-step flag variety for $s<r$ admits a rank 1 point, then $X=F\left(d_{1}, . ., d_{r}, n\right)$ admits one as well. 
Case 6. Assume $n=2 d_{a}$ with $r>a>1$. Consider

$$
\begin{aligned}
& X_{1}=F\left(d_{1}, . ., d_{a}\right) \equiv F\left(d_{1}, . ., d_{a}, d_{a}\right) \hookrightarrow F\left(d_{1}, . ., d_{a}, n\right),\left(E^{\bullet}\right) \mapsto\left(0_{n-d_{a}} \oplus E^{\bullet}\right) \\
& X_{2}=F\left(d_{a+1}-d_{a}, . ., d_{r}-d_{a}, n-d_{a}\right) \hookrightarrow F\left(d_{a+1}, . ., d_{r}, n\right),\left(E^{\bullet}\right) \mapsto\left(E^{\bullet} \oplus \mathbb{C}^{d_{a}}\right)
\end{aligned}
$$

Here we view $\mathbb{C}^{n}=\mathbb{C}^{n-d_{a}} \oplus \mathbb{C}^{d_{a}}$. Let $M_{1}, M_{2}, M$ be the Stiefel bundles over $X_{1}, X_{2}, X$ respectively. Let $f_{1}, f_{2}$ be rank 1 points of $X_{1}, X_{2}$ respectively with the hyperplane properties

$$
\left(x_{J_{1}} \cdots x_{J_{a}}\right)\left|f_{1},\left(x_{J_{a+1}^{\prime}} \cdots x_{J_{r}^{\prime}}\right)\right| f_{2}
$$

for some $J_{i} \subset\left(1,2, . ., n-d_{a}\right)$ with $\left|J_{i}\right|=d_{i}(i=1, \ldots, a-1)$ and $J_{a-1}=\left(d_{a}-d_{a-1}+1, . ., d_{a}\right)$, and for some $J_{i}^{\prime} \subset\left(1,2, . ., n-d_{a}\right)$ with $\left|J_{i}^{\prime}\right|=d_{i}-d_{a}(i=a+1, . ., r)$ and $J_{r}^{\prime}=\left(n-d_{r}+\right.$ $\left.1, . ., n-d_{a}\right)$. Put $J:=\left(n-d_{a}+1, . ., n\right), J_{i}:=J_{i}^{\prime} \cup J, i=a+1, . ., n$, and

$$
f:=\bar{f}_{1} \cdot \tilde{f}_{2} \cdot\left(x_{J}\right)^{d_{a+1}-d_{a-1}-1}\left(x_{1, \ldots, d_{a}}\right) .
$$

Then $f$ has the hyperplane property

$$
\left(x_{J_{1}} \cdots \widehat{x_{J_{a}}} \cdots x_{J_{r}} x_{J}\right) \mid f .
$$

Lemma 8.12. The special section $m=\left(m_{r}, . ., m_{1}\right)$ (cf. Lemma 8.1) of $M(f) \rightarrow X(f)$ has the following form:

$$
\begin{aligned}
m_{i} & =\left[\begin{array}{cc}
m_{i}^{\prime} & A_{i} \\
O & I_{d_{a}}
\end{array}\right], i=a+1, . ., r \\
m_{a} & =\left[\begin{array}{c}
A_{a} \\
I_{d_{a}}
\end{array}\right] \\
m_{r} \cdots m_{a} & =\left[\begin{array}{c}
D \\
I_{d_{a}}
\end{array}\right] \\
m_{a-1} & =D^{-1} m_{a-1}^{\prime} \\
m_{i} & =m_{i}^{\prime}, \quad i=1, . ., a-2
\end{aligned}
$$

where $D$ is a $G L_{d_{a}}$-valued function, $A_{a}, . ., A_{r}$ are matrix valued functions, and $\left(m_{a-1}^{\prime}, . ., m_{1}^{\prime}\right)$, $\left(m_{r}^{\prime}, . ., m_{a+1}^{\prime}\right)$ are matrix valued functions taking values in the special sections of the $M_{1}\left(f_{1}\right) \rightarrow$ $X_{1}\left(f_{1}\right), M_{2}\left(f_{2}\right) \rightarrow X_{2}\left(f_{2}\right)$ respectively.

The proof is a degenerate version of the lemma in Case 5 (with $m_{a}^{\prime}$ missing but with $\left(1, . ., d_{a}\right)$ play the role of $\left.J_{a}\right)$, and will be omitted. The lemma and Theorem 1.4 imply

Proposition 8.13. For $n=2 d_{a}$ with $r>a>1$, if any s-step flag variety for $s<r$ admits a rank 1 point $f$ with the hyperplane property, then $X=F\left(d_{1}, . ., d_{r}, n\right)$ admits one as well.

Case 7. Assume $n=d_{a-1}+d_{a}$ with $r>a>1$. If $a=2$ then it is Case 4 , so we can assume $a \geq 3$ (and $r \geq 4$ ). Consider

$$
\begin{aligned}
& X_{1}:=F\left(d_{1}, . ., d_{a-2}, d_{a-1}\right) \hookrightarrow F\left(d_{1}, . ., d_{a-2}, n\right), E^{\bullet} \mapsto E^{\bullet} \oplus 0_{n-d_{a-1}} \\
& X_{2}:=F\left(d_{a-1}, . ., d_{r}, n\right) .
\end{aligned}
$$


Here we view $\mathbb{C}^{n}=\mathbb{C}^{d_{a-1}} \oplus \mathbb{C}^{n-d_{a-1}}$. Let $M_{1}, M_{2}, M$ be the Stiefel bundles over $X_{1}, X_{2}, X$ respectively. Let $f_{1}, f_{2}$ be rank 1 points of $X_{1}, X_{2}$ respectively with the hyperplane properties

$$
\left(x_{J_{1}} \cdots x_{J_{a-2}}\right)\left|f_{1},\left(\left(x_{J_{a-1}}\right)^{d_{a-1}} x_{J_{a}} \cdots x_{J_{r}}\right)\right| f_{2}
$$

for some $J_{i} \subset\left(1,2, . ., d_{a-1}\right)$ with $\left|J_{i}\right|=d_{i}(i=1, \ldots, a-2)$, and for some $J_{i} \subset(1,2, . ., n)$ with $\left|J_{i}\right|=d_{i}(i=a-1, . ., r)$ and $J_{a-1}=\left(1, . ., d_{a-1}\right)$. Note that such an $f_{2}$ exists by Proposition 8.7 in Case 4, if any $s$-step flag variety for $s<r$ admits a rank 1 point with the hyperplane property.

Put

$$
f=\bar{f}_{1} \cdot \bar{f}_{2} \cdot\left(x_{J_{a-1}}\right)^{-d_{a-2}} \in \Gamma\left(X, \omega_{X}^{-1}\right) .
$$

Then $f$ has the hyperplane property

$$
\left(x_{J_{1}} \cdots x_{J_{r}}\right) \mid f
$$

Lemma 8.14. We have an $H=G L_{d_{r}} \times \cdots \times G L_{d_{1}}$ equivariant isomorphism

$$
\begin{aligned}
& M_{1}\left(f_{1}\right) \times M_{2}\left(f_{2}\right) \rightarrow M(f) \\
& \left(m_{a-2}^{\prime}, . ., m_{1}^{\prime}\right),\left(m_{r}^{\prime}, . ., m_{a-1}^{\prime}\right) \mapsto m=\left(m_{r}^{\prime}, . ., m_{a-1}^{\prime}, D^{-1} m_{a-2}^{\prime}, m_{a-3}^{\prime}, . ., m_{1}^{\prime}\right)
\end{aligned}
$$

where $D$ is the $J_{a-1}$-block of $m_{r}^{\prime} \cdots m_{a-1}^{\prime}$. Hence the map descends to an isomorphism

$$
X_{1}\left(f_{1}\right) \times X_{2}\left(f_{2}\right) \rightarrow X(f) .
$$

The proof is almost identical to the lemmas in Cases 1 and 3, and will be omitted. The lemma and Theorem 1.4 imply

Proposition 8.15. For $d_{a-1}+d_{a}=n$ with $r>a>1$, if any $s$-step flag variety for $s<r$ admits a rank 1 point with the hyperplane property, then $X=F\left(d_{1}, . ., d_{r}, n\right)$ admits one as well.

Now combining the propositions in all Cases 1-7 yields a complete recursive procedure for constructing a rank 1 point with the hyperplane property for any $r$-step flag variety, proving Corollary 1.6

Example 8.16. Consider $X=F(1,2,3,5)$, which belongs in Case 3. Let $X_{1}=F(1,2)$ and take $f_{1}=x_{1} x_{2}$. Let $X_{2}=F(2,3,5)$, which belongs in Case 2, and we can take $f_{2}=\left(x_{12}\right)^{3}\left(x_{345}\right)^{3}$ as a rank 1 point of $X_{2}$, by Proposition 8.4 Therefore,

$$
f=x_{1} x_{2}\left(x_{12}\right)^{3}\left(x_{345}\right)^{3}\left(x_{12}\right)^{-1}
$$

is rank 1 point of $X$ according to the construction in Case 3 . 
Example 8.17. Consider the flag variety of $S L_{5}, X=F(1,2,3,4,5)$, which belongs in Case 7 with $a=3$. Let $X_{1}=F(1,2)$ and take $f_{1}=x_{1} x_{2}$. Let $X_{2}=F(2,3,4,5) \cong F(1,2,3,5)$, which is the preceding example. Applying this isomorphism to the rank 1 point there, we get $f_{2}=x_{2345} x_{1345}\left(x_{345}\right)^{2}\left(x_{12}\right)^{3}$ as a rank 1 point of $X_{2}$. Therefore,

$$
f=x_{1} x_{2} x_{2345} x_{1345}\left(x_{345}\right)^{2}\left(x_{12}\right)^{3}\left(x_{12}\right)^{-1}
$$

is a rank 1 point of $X$ according to the construction in Case 7 .

\section{Appendix A. Theory of $D$-Modules}

We recall the theory of algebraic $D$-modules. A standard reference is $[5]$.

Let $X$ be an algebraic variety over $k$ of characteristics zero. Let $\operatorname{Hol}\left(D_{X}\right)$ be the category of holonomic (left) $D$-modules on $X$. Its bounded derived category is denoted by $D_{h}^{b}(X)$.

Let $f: X \rightarrow Y$ be a morphism, there are the following pairs of adjoint (derived) functors (following the notation of Borel's book)

$$
f^{+}: D_{h}^{b}(Y) \rightleftharpoons D_{h}^{b}(X): f_{+}, \quad f_{!}: D_{h}^{b}(X) \rightleftharpoons D_{h}^{b}(Y): f^{!} .
$$

Recall the definition of $f_{+}$in the following cases (assuming $X$ and $Y$ are smooth): in the case, there is an $f^{-1} D_{Y} \times D_{X}$-bimodule $D_{Y \leftarrow X}$ on $X$, and

$$
f_{+}(M)=R f_{*}\left(D_{Y \leftarrow X} \otimes^{L} M\right) .
$$

Without mentioning the exact definition of this bimodule $D_{Y \leftarrow X}$, we concentrate on the following special cases. Let $d_{X, Y}=\operatorname{dim} X-\operatorname{dim} Y$.

(i) $f: X \rightarrow Y$ is smooth. Then $f_{+}$(up to shift) is the usual construction of the GaussManin connection. I.e.

$$
f_{+}(M)=R f_{*}\left(M \otimes \Omega_{X / Y}^{\bullet}\left[d_{X, Y}\right]\right) .
$$

In particular, $H^{i} f_{+} \mathcal{O}_{X}$ is the $D$-module on $Y$ formed by the $\left(i+d_{X, Y}\right)$ th relative De Rham cohomology. In particular, if $f$ is an open embedding, then $f_{+}(M)=R f_{*} M$ as quasicoherent sheaves on $Y$. Observe that under the this normalization of the cohomological degrees, $H^{0} f_{+} \mathcal{O}_{X}$ is the usual "middle dimension" cohomology of the family $f: X \rightarrow Y$.

Example A.1. A particular example: $j: \mathbb{G}_{m}=\operatorname{Spec} k\left[x, x^{-1}\right] \rightarrow \mathbb{A}^{1}=\operatorname{Spec} k[x]$ the open embedding. Then $j_{+} \mathcal{O}_{\mathbb{G}_{m}}$ as a $D$-module on $\mathbb{A}^{1}$ is isomorphic to $k\left[x, \partial_{x}\right] /\left(x \partial_{x}+1\right)$.

(ii) $f: X \rightarrow Y$ is a closed embedding given by the ideal $\mathcal{I}$. Then

$$
f_{+}(M)=f_{*}\left(D_{Y} / D_{Y} \mathcal{I} \otimes \omega_{X / Y} \otimes M\right)
$$

where $\omega_{X / Y}$ is the relative canonical sheaf $\omega_{X / Y}=\omega_{X} \otimes\left(\left.\omega_{Y}^{-1}\right|_{X}\right)$. 
Example A.2. A particular example: let $Y$ be $\mathbb{A}^{n}=\operatorname{Spec} k\left[x_{1}, \ldots, x_{n}\right]$ and $i: X \rightarrow Y$ be the inclusion of the vector space given by $x_{1}=\cdots=x_{r}=0$. Then $x_{r+1}, \ldots, x_{n}$ form a coordinate system on $X$. Let $M=\mathcal{O}_{X}=D_{X} / D_{X}\left(\partial_{r+1}, \ldots, \partial_{n}\right)$. Then

$$
i_{+} M=D_{Y} / D_{Y}\left(x_{1}, \ldots, x_{r}, \partial_{r+1}, \ldots, \partial_{n}\right),
$$

called the delta sheaf supported on $X$, denoted by $\delta_{X}$.

Observe that there is the following exact sequence of $D_{\mathbb{A}^{1}}$-modules

$$
0 \rightarrow \mathcal{O}_{\mathbb{A}^{1}} \rightarrow j_{+} \mathcal{O}_{\mathbb{G}_{m}} \rightarrow \delta_{\{0\}} \rightarrow 0 .
$$

Next, we recall the definition of $f^{!}$. There is a $D_{X} \times f^{-1} D_{Y}$-bimodule $D_{X \rightarrow Y}$ on $X$, and by definition

$$
f^{!}(M)=D_{X \rightarrow Y} \otimes_{f^{-1} D_{Y}}^{L} f^{-1} M\left[d_{X, Y}\right] .
$$

As quasi-coherent $\mathcal{O}_{X}$-modules,

$$
f^{!}(M)=L f^{*} M\left[d_{X, Y}\right]
$$

Again, let us mention the following special cases.

(i) $f: X \rightarrow Y$ is smooth. In this case, $f^{!}\left[-d_{X, Y}\right]$ is exact, and as quasi-coherent sheaves, $f^{!}\left[-d_{X, Y}\right](M)=f^{*} M$. In particular, if $f$ is an open embedding, then $f^{!} M=\left.M\right|_{X}$.

(ii) $f: X \rightarrow Y$ is a closed embedding, given by the ideal sheaf $\mathcal{I}$. In this case

$$
H^{0} f^{!}(M) \otimes \omega_{X / Y}=\{m \in M \mid x m=0 \text { for any } x \in \mathcal{I}\} .
$$

The following distinguished triangle generalizes A.1): Let $i: X \rightarrow Y$ be a closed embedding and $j: U \rightarrow Y$ be the complement.

$$
i_{+} i^{!} M \rightarrow M \rightarrow j_{+} j^{!} M \rightarrow
$$

Indeed, in the case $Y=\mathbb{A}^{1}$ and $X=\mathbb{G}_{m}, M=\mathcal{O}_{\mathbb{A}^{1}}$, we recover A.1.

The following theorem (Kashiwara's lemma) is of fundamental importance,

Theorem A.3. Let $i: X \rightarrow Y$ be a closed embedding.

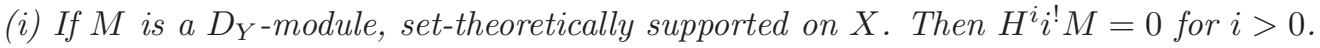

(ii) Let $D_{Y}-\operatorname{Mod}_{X}$ be the category of $D_{Y}$-modules, set-theoretically supported on $X$, and $D_{X}$-Mod be the category of $D_{X}$-modules. Then there is an equivalence of categories

$$
i_{+}: D_{X}-\operatorname{Mod} \rightleftharpoons D_{Y}-\operatorname{Mod}_{X}: H^{0} i^{!} .
$$


In the sequel, we will make use of the following notation: let $i: X \rightarrow Y$ be a locally closed embedding. If $M$ is a D-module on $Y$, set-theoretically supported on $\bar{X}$, then $H^{0} i^{!} M$ will be denoted by $\left.M\right|_{X}$.

This finishes the discussion of the functors $f_{+}, f$ ! Then $f_{!}$is defined to be the left adjoint of $f^{!}$and $f^{+}$is defined to be the left adjoint of $f_{+}$. Recall that there is the duality functor $\mathbb{D}_{X}: D_{h}^{b}(X) \rightarrow D_{h}^{b}(X)$. We can also express $f^{+}=\mathbb{D}_{X} f^{!} \mathbb{D}_{Y}$ and $f_{!}=\mathbb{D}_{Y} f_{+} \mathbb{D}_{X}$. It is known that

(i) If $f: X \rightarrow Y$ is a closed embedding (or more generally if $f$ is proper), $f_{!}=f_{+}$.

(ii) If $f: X \rightarrow Y$ is an open embedding, $f^{!}=f^{+}$.

Remark A.1. The definitions of $f_{+}, f^{!}$do not require the holonomicity, and therefore they are defined on the whole category of (not necessarily holonomic) $D$-modules. However, as functors on the whole category of $D$-modules, they do not admit adjoint functors and therefore $f_{!}, f^{+}$are not defined in general.

Example A.4. Let $j: \mathbb{G}_{m} \rightarrow \mathbb{A}^{1}$ as before. One can show that $j_{!} \mathcal{O}_{\mathbb{G}_{m}} \simeq k[x, \partial] / x \partial$.

The dual version of A.1 is

$$
0 \rightarrow \delta_{\{0\}} \rightarrow j_{!} \mathcal{O}_{\mathbb{G}_{m}} \rightarrow \mathcal{O}_{\mathbb{A}^{1}} \rightarrow 0,
$$

and the dual version of (A.2) is

$$
j ! j^{!} M \rightarrow M \rightarrow i_{+} i^{+} M \rightarrow
$$

Now let $k=\mathbb{C}$. Let $D_{r h}^{b}(X)$ be the bounded derived category of holonomic $D$-modules with regular singularities, and let $D_{c}^{b}\left(X^{a n}\right)$ be the bounded derived category of constructible sheaves on $X^{a n}$ (we denote $X$ equipped with the classical topology by $X^{a n}$ ). Then RiemannHilbert correspondence is an equivalence

$$
\mathrm{RH}: D_{r h}^{b}(X) \simeq D_{c}^{b}(X), \quad \mathrm{RH}(M)=\omega_{X^{a n}} \otimes^{L} M^{a n}=\Omega_{X^{a n}}^{\bullet} \otimes M^{a n}[\operatorname{dim} X],
$$

where $\omega_{X^{a n}}$ is the canonical sheaf on $X^{a n}$, regarded as a right $D$-module via Lie derivative, and the derived tensor product is over $D_{X^{a n}}$. This correspondence is compatible with the six operation functors. In particular,

$$
\mathrm{RH} f_{+} \simeq f_{*} \mathrm{RH}, \quad \mathrm{RH} f_{!} \simeq f_{!} \mathrm{RH}, \quad \mathrm{RH} f^{!} \simeq f^{!} \mathrm{RH}, \quad \mathrm{RH} f^{+} \simeq f^{*} \mathrm{RH} .
$$

If $M$ is a plain $D$-module, then $\operatorname{RH}(M)$ is a perverse sheaf on $X^{a n}$. While the above equivalence is covariant, sometimes one also consider the contravariant version

$$
\text { Sol : } D_{r h}^{b}(X) \simeq D_{c}^{b}(X)^{o p}, \quad \operatorname{Sol}(M)=R \operatorname{Hom}_{D_{X^{a n}}}\left(M^{a n}, \mathcal{O}_{X^{a n}}\right) .
$$


The relation between Sol and $\mathrm{RH}$ is $\mathrm{RH}=\operatorname{Sol} \mathbb{D}_{X}[\operatorname{dim} X]$.

Next, we discuss background materials on equivariant D-modules, most which can be found in [5] [11]. Let $G$ be a connected algebraic group and $\mathfrak{g}=\operatorname{Lie} G$. Let us regard $\mathfrak{g}$ as right invariant vector fields on $G$, and for a Lie algebra homomorphism $\chi: \mathfrak{g} \rightarrow k$, we define a character D-module on $G$ by

$$
\mathcal{L}_{\chi}=D_{G} / D_{G}(\xi+\chi(\xi), \xi \in \mathfrak{g}) .
$$

This is a rank one local system on $G$. In particular, it is holonomic. It is called a character sheaf because if we denote by mult : $G \times G \rightarrow G$ the multiplication map of $G$, then there is a canonical isomorphism mult ${ }^{!} \mathcal{L}_{\chi} \simeq \mathcal{L}_{\chi} \otimes \mathcal{L}_{\chi}[\operatorname{dim} G]$ satisfying the cocycle condition under the further !-pullback to $G \times G \times G$.

Let $Z$ be a $G$-variety and act : $G \times Z \rightarrow Z$ be the action map. A $(G, \chi)$-equivariant, or a $G$-monodromic against $\chi, \mathrm{D}$-module on $Z$ is a D-module on $Z$ together with an isomorphism

$$
\theta: \operatorname{act} ! M \simeq \mathcal{L}_{\chi} \otimes M[\operatorname{dim} G],
$$

satisfying the usual cocycle condition under the further !-pullback to $G \times G \times Z$.

The following lemma is well-known, which can be proved as in [5, Theorem 12.11]. See also [11, §II.5].

Lemma A.5. Assume that there are only finitely many orbits under the action of $G$ on $Z$, then any $(G, \chi)$-equivaraint $D$-module is holonomic. In addition, if $\mathcal{L}_{\chi}$ is regular singular, then any $(G, \chi)$-equivariant $D$-module is regular singular.

We will need the following lemma. Let $U \mathfrak{g}$ be the universal enveloping algebra of $\mathfrak{g}$. Then $\chi$ defines a one-dimensional $U \mathfrak{g}$-module, denoted by $k_{\chi}$. Note that if $Z$ is a $G$-variety, we have the corresponding infinitesimal action $d a: \mathfrak{g} \rightarrow T_{Z}$, which extends to $U \mathfrak{g} \rightarrow D_{Z}$.

Lemma A.6. The D-module

$$
D_{Z, \chi}=D_{Z} / D_{Z}(d a(\xi)+\chi(\xi), \xi \in \mathfrak{g})=\left(D_{Z} \otimes k_{\chi}\right) \otimes_{U \mathfrak{g}} k
$$

is a natural $(G, \chi)$-equivariant $D$-module on $Z$.

More generally, note that $D_{Z}$ is naturally $G$-equivariant as $\mathcal{O}$-modules, i.e., there is an isomorphism of $\mathcal{O}$-modules $\theta:$ act* $D_{Z} \simeq p_{Z}^{*} D_{Z}$ satisfying the cocycle condition. Let $I \subset D_{Z}$ be a $G$-invariant left ideal, then

$$
D_{Z} / I+D_{Z}(d a(\xi)+\chi(\xi), \xi \in \mathfrak{g})
$$

is $(G, \chi)$-equivariant. 
Note that in the above lemma, we do not need to assume that $G$ acts on $Z$ with finitely many orbits. See [11, §II.3].

Note that if $i: H \rightarrow G$ is a connected closed subgroup, $i ! \mathcal{L}_{\chi}[-\operatorname{dim} H]=D_{H} / D_{H}(\xi+$ $\chi(\xi), \xi \in \mathfrak{h})=\mathcal{L}_{\left.\chi\right|_{\mathfrak{h}}}$. We have the following simple observation.

Lemma A.7. Let $Z=G / H$ be a homogeneous $G$-variety. Let $\chi: \mathfrak{g} \rightarrow k$ be a Lie algebra homomorphism and $\mathcal{L}_{\chi}$ be the rank character $D$-module on $G$ as in A.5. Then if $\mathcal{L}_{\chi \mid \mathfrak{h}} \neq$ $\mathcal{O}_{H^{\circ}}$, where $H^{\circ}$ is the neutral connected component of $H$, there is no D-module on $Z$, equivariant with respect to $G$ against $\chi$.

Proof. Let $M$ be a non-zero $(G, \chi)$-equivariant D-modules on $Z$. Let $i: H^{\circ} \rightarrow G$ be the inclusion, and $i_{e}: e H \rightarrow Z$ be the inclusion of the identity coset. Consider the diagram

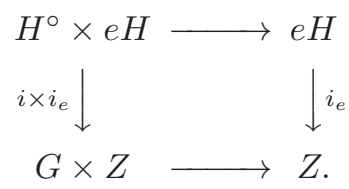

Then $i^{!} \mathcal{L}_{\chi} \otimes i_{e}^{!} M=\left(i \times i_{e}\right) !$ act! $M=\mathcal{O}_{H} \otimes i_{e}^{!} M[\operatorname{dim} Z]$. Therefore, $\mathcal{L}_{\left.\chi\right|_{\mathfrak{h}}}=\mathcal{O}_{H}$.

Example A.8. Let $\lambda \in k^{\times}$, and let $\mathcal{L}_{\lambda}$ be the $D$-module on $\mathbb{G}_{m}$ given by $x \partial+\lambda$. I.e. $\mathcal{L}_{\lambda}$ is the local system on $\mathbb{G}_{m}$ with monodromy $\exp (-2 \pi \sqrt{-1} \lambda$ ) (via the Riemann-Hilbert correspondence if $k=\mathbb{C}$ ). This is a character D-module on $G$ with $\chi(x \partial)=\lambda$. If $\lambda \in \mathbb{Z}$, then $\mathcal{L}_{\lambda} \simeq \mathcal{O}_{\mathbb{G}_{m}}$. Let $j: \mathbb{G}_{m} \rightarrow \mathbb{A}^{1}$ be the open embedding. Then both $j_{+} \mathcal{L}_{\lambda}$ and $j_{!} \mathcal{L}_{\lambda}$ are $(G, \lambda)$-equivaraint D-modules on $\mathbb{G}_{m}$. If $\lambda$ is not an integer, then $j_{!} \mathcal{L}_{\lambda} \simeq j_{+} \mathcal{L}_{\lambda}$. In this case, this $D$-module is irreducible on $\mathbb{A}^{1}$.

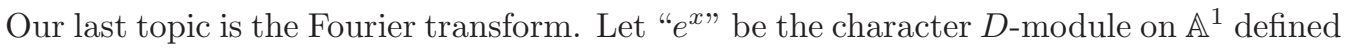
by $\partial-1$. Let $V$ be a vector space and $V^{\vee}$ be its dual. We have the natural pairing

$$
m: V \times V^{\vee} \rightarrow \mathbb{A}^{1} .
$$

The pullback of $e^{x}$ along $m$ is still denoted by $e^{x}$. Let $p_{V}, p_{V^{\vee}}$ be the projections of $V \times V^{\vee}$ to the two factors. The Fourier transform is defined as

$$
\mathcal{F} \text { our }(M)=p_{V^{\vee},+}\left(p_{V}^{!}(M) \otimes e^{x}\right)[\operatorname{dim} V],
$$

Fourier transform $\mathcal{F}$ our is an exact functor, and can be described in the following simple way. Let $M$ be a $D$-module on $V$, and therefore is identified with a module over the Weyl algebra $k\left[a_{1}, \ldots, a_{n}, \partial_{a_{1}}, \ldots, \partial_{a_{n}}\right]$. Then $\mathcal{F}$ our $(M)$ as a vector space is identified with $M$, and the $D$-module structure is given by $a_{i}^{*} m=\partial_{a_{i}} m$ and $\partial_{a_{i}^{*}}=-a_{i} m$. In other words, if we denote the ring homomorphism

$$
\text { ^: } D_{V} \rightarrow D_{V^{\vee}}, \quad \widehat{a_{i}}=-\partial_{a_{i}^{*}}, \widehat{\partial_{a_{i}}}=a_{i}^{*},
$$

then $\mathcal{F}$ our $(M)=D_{V^{\vee}} \otimes_{D_{V}} M$. See [6, p85]. 
Example A.9. Let $W \subset V$ be a vector subspace, and $W^{\perp}$ be the orthogonal complement of $W$ in $V^{\vee}$. Then $\mathcal{F}$ our $\left(\delta_{W}\right)=\delta_{W^{\perp}}$.

Example A.10. More generally, let $i: W \subset V$ be a vector subspace, and $0 \rightarrow W^{\perp} \rightarrow V^{\vee} \stackrel{p}{\rightarrow}$ $W^{\vee} \rightarrow 0$ be the dual sequence. Let $M$ be a D-module on $W$. Then

$$
\mathcal{F} \text { our }\left(i_{+} M\right)=p ! \mathcal{F} \text { our }(M)[\operatorname{dim} W-\operatorname{dim} V] .
$$

Example A.11. Let $V=\mathbb{A}^{1}$ and we identify $V^{\vee}=\mathbb{A}^{1}$ via the natural multiplication $\mathbb{A}^{1} \times$ $\mathbb{A}^{1} \rightarrow \mathbb{A}^{1}$. Then under the Fourier transform, the exact sequence (A.1) becomes (A.3).

Example A.12. Recall the character D-module $\mathcal{L}_{\lambda}$ on $\mathbb{G}_{m}$. Let $j: \mathbb{G}_{m} \rightarrow \mathbb{A}^{1}$ be the open immersion. Then

$$
\mathcal{F} \text { our }\left(j_{+} \mathcal{L}_{\lambda}\right)=j_{!} \mathcal{L}_{-\lambda+1}
$$

Fourier transform preserves holonomicity. If $M$ is holonomic, the we can also write

$$
\mathcal{F} \text { our }(M)=p_{V^{\vee}, !}\left(p_{V}^{*}(M) \otimes e^{x}\right)[-\operatorname{dim} V] .
$$

However, Fourier transform does not necessarily preserves the regular singularity. For example, the Fourier transform of the delta sheaf on $\mathbb{A}^{1}$ supported at $1 \in \mathbb{A}^{1}(k)$ is $e^{x}$. However, under certain circumstance, one can show that $\mathcal{F}$ our $(M)$ is regular singular. Let $\mathbb{G}_{m}$ act on $V$ via homotheties, i.e. mult : $\mathbb{G}_{m} \times V \rightarrow V$, mult $(a, v)=a v$. Let $\lambda: \operatorname{Lie} \mathbb{G}_{m} \rightarrow k$ be a map. Recall the notion of $\left(\mathbb{G}_{m}, \lambda\right)$-equivariant D-modules. We say a holonomic $D$-module on $V$ to be $\mathbb{G}_{m}$ monodromic if each of its irreducible constitutes is $\left(\mathbb{G}_{m}, \lambda\right)$-equivariant for some $\lambda$. Observe that $e^{x}$ is not $\mathbb{G}_{m}$-monodromic.

Let $D_{r h, m}^{b}(V)$ be the full subcategory of $D_{r h}^{b}(V)$ whose cohomology sheaves are regular holonomic and $\mathbb{G}_{m}$-monodromic.

Lemma A.13. The Fourier transform restricts to an equivalence

$$
\mathcal{F} \text { our : } D_{r h, m}^{b}(V) \simeq D_{r h, m}^{b}\left(V^{\vee}\right) .
$$

Proof. [6, Theorems 7.4, 7.24].

Fourier transform can be generalized to family versions. Let $X$ be a base variety, and $\mathbb{V}$ a vector bundle over $X, \mathbb{V}^{\vee}$ the dual bundle, so there is

$$
m: \mathbb{V} \times{ }_{X} \mathbb{V}^{\vee} \rightarrow \mathbb{A}^{1} .
$$

Then one can define

$$
\mathcal{F}_{\operatorname{our}}(M)=p_{\mathbb{V} \vee},+\left(p_{\mathbb{V}}^{!}(M) \otimes e^{x}\right)[\mathrm{rk} \mathbb{V}]
$$


Note that the family version of Example A.10 still holds. More precisely, let $i: \mathbb{W} \subset \mathbb{V}$ be a subbundle on $p: \mathbb{V}^{\vee} \rightarrow \mathbb{W}^{\vee}$ be the dual map. Then

$$
\mathcal{F}_{\text {our }}\left(i_{+} M\right)=p ! \mathcal{F} \operatorname{our}_{X}(M)[\mathrm{rk} \mathbb{W}-\mathrm{rk} \mathbb{V}] \text {. }
$$

Note that family version of Fourier transforms commute with base change. Namely, if $f: Y \rightarrow X$ is a map. Then $\mathcal{F} \operatorname{our}_{Y}\left(f^{!} M\right)=f^{!} \mathcal{F} \operatorname{our}_{X}(M)$.

Let us consider the family version of Example A.12. So we assume that $\mathbb{V}=\mathbb{L}$ is a line bundle, on which $\mathbb{G}_{m}$ acts by homotheties. Let $\mathbb{L}=\mathbb{L}-X$, where $X$ is regarded as the zero section of $\mathbb{L}$. Let $\mathbb{L}^{\vee}$ be the dual vector bundle of $\mathbb{L}$ and $\mathbb{L}^{\vee}$ is defined similarly. Let $M$ be a $\left(\mathbb{G}_{m}, \lambda\right)$-equivariant D-module on $\mathbb{L}$.

The following lemma is useful.

Lemma A.14. Let $X$ be proper and $\mathbb{V}=X \times V$ be the trivial bundle over $X$. Let $\pi$ : $X \times V \rightarrow V$ and $\pi^{\vee}: X \times V^{\vee} \rightarrow V^{\vee}$ be the projections. Then

$$
\mathcal{F} \text { our } \circ \pi_{!} \simeq \pi_{!}^{\vee} \circ \mathcal{F}_{\text {our }}^{X} \text {. }
$$

Proof. This follows from the base change theorem for D-modules (cf. [5, VI, §8]). Namely, as $X$ is proper, $\pi_{+}=\pi_{!}$, etc. We have the following commutative diagrams with both squares Cartesian.

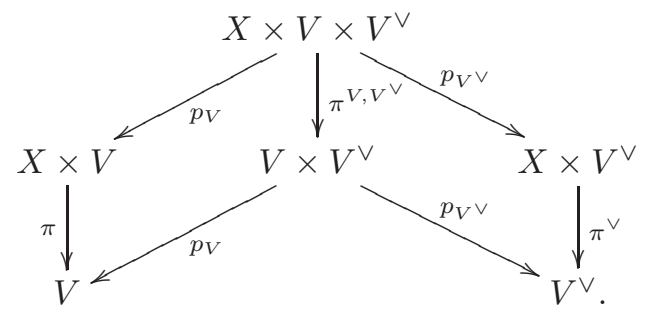

Then

$$
\begin{aligned}
\mathcal{F} \text { our }\left(\pi_{!}(M)\right) & =p_{V^{\vee},+}\left(p_{V}^{!} \pi_{+}^{\vee}(M) \otimes e^{x}\right) \\
& =p_{V^{\vee},+}\left(\pi_{+}^{V, V} p_{V}^{!}(M) \otimes e^{x}\right) \\
& =p_{V^{\vee},+}^{V, V_{+}^{\vee}}\left(p_{V}^{!}(M) \otimes e^{x}\right) \\
& =\pi_{+}^{\vee} \mathcal{F}^{\text {our }_{X}}(M)
\end{aligned}
$$

\section{REFERENCES}

[1] A. Adolphson, Hypergeometric Functions and Rings Generated by Monomials, Duke Math. J. Vol 73, No. 2 (1994) 269-290.

[2] J. Bernstein, I. M. Gelfand, S. I. Gelfand, A certain category of g-modules, Functional Analysis and its Applications. 10 no. 2 (1976), 1-8.

[3] A. Beilinson, V. Ginzburg, W. Soergel, Koszul duality patterns in Representation Theory, Journ. AMS 9 (1996), 473-527. 
[4] S. Bloch, A. Huang, B.H. Lian, V. Srinivas, and S.-T. Yau, On the Holonomic Rank Problem, arXiv:1302.4481v1.

[5] A. Borel et al, Algebraic D-modules, Academic Press 1987.

[6] J.-L. Brylinski, Transformations canoniques, dualité projective, théorie de Lefschetz, trans- formations de Fourier et sommes trigonométriques, Astérisque 140-141 (1986), 3134.

[7] I. Gel'fand, M. Kapranov and A. Zelevinsky, Hypergeometric functions and toral manifolds, English translation, Functional Anal. Appl. 23 (1989), 94-106.

[8] C. Hague, On the B-canonical splittings of flag varieties, J. Algebra 323 (2010), no. 6, 17581764. arXiv:0908.4354.

[9] S. Hosono, B.H. Lian and S-T. Yau, Maximal degeneracy points of GKZ systems, Journ. AMS Vol. 10, No. 2 (1997) 427-443.

[10] S. Hosono, B.H. Lian and S-T. Yau, GKZ-generalized hypergeometric systems in mirror symmetry of Calabi-Yau hypersurfaces, Commun. Math. Phys. 182 (1996), 535-577.

[11] R. Hotta, Equivariant D-modules, arXiv:math/9805021v1.

[12] M. Kapranov, Hypergeometric functions on reductive groups, Integrable systems and algebraic geometry (Kobe/Kyoto, 1997), 236-281, World Sci. Publ., River Edge, NJ, 1998.

[13] A. Knutson, T. Lam and D. Speyer, Projections of Richardson Varieties, arXiv:1008.3939v2.

[14] A. Knutson, T. Lam and D. Speyer, Positroid Varieties: Juggling and Geometry, arXiv:1111.3660v1.

[15] G. Laumon, Transformation de Fourier homogène Bull. Soc. math. France 131 (4), 2003, p.527-551.

[16] B.H. Lian, R. Song and S.-T. Yau, Period Integrals and Tautological Systems, arXiv:1105.2984v3, to appear in Journ. EMS 2013.

[17] B.H. Lian and S.-T. Yau, Period Integrals of CY and General Type Complete Intersections, Invent. Math. Vol 191, 1 (2013) 35-89. arXiv:1105.4872v3.

[18] G. Lusztig, Total Positivity in Partial Flag Manifolds, Representation Theory, AMS, Vol. 2 (1998) 7078.

[19] R. Virk, Extensions of Verma Modules, http://math.colorado.edu/ ravi1033/pub/verma.pdf.

[20] K. Reitsch, Closure Relations for Totally Nonnegative Cells in G/P, arXiv:0509137v2.

A. Huang, Department of Mathematics, Harvard University, Cambridge MA 02138. anhuang@math.harvard.edu.

B.H. Lian, Department of Mathematics, Brandeis University, Waltham MA 02454. lian@brandeis.edu.

X. Zhu, Department of Mathematics, Northwestern University, Evanston IL 60208. xinwenz@math.northwestern.edu. 